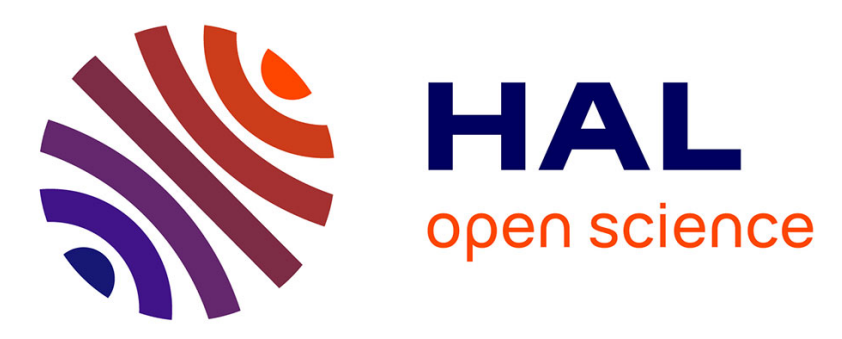

\title{
Finite element methods for the temperature in composite media with contact resistance
}

Faker Ben Belgacem, Christine Bernardi, Faten Jelassi, Maimouna Mint Brahim

\section{- To cite this version:}

Faker Ben Belgacem, Christine Bernardi, Faten Jelassi, Maimouna Mint Brahim. Finite element methods for the temperature in composite media with contact resistance. 2014. hal-01005515

\section{HAL Id: hal-01005515 https: / hal.sorbonne-universite.fr/hal-01005515}

Submitted on 13 Jun 2014

HAL is a multi-disciplinary open access archive for the deposit and dissemination of scientific research documents, whether they are published or not. The documents may come from teaching and research institutions in France or abroad, or from public or private research centers.
L'archive ouverte pluridisciplinaire $\mathbf{H A L}$, est destinée au dépôt et à la diffusion de documents scientifiques de niveau recherche, publiés ou non, émanant des établissements d'enseignement et de recherche français ou étrangers, des laboratoires publics ou privés. 


\title{
Finite element methods for the temperature in composite media with contact resistance
}

\author{
by Faker Ben Belgacem ${ }^{1}$, Christine Bernardi ${ }^{2}$, \\ Faten Jelassi ${ }^{3,4}$ and Maimouna Mint Brahim ${ }^{3}$
}

\begin{abstract}
We consider a heat diffusion problem inside a composite medium. The contact resistance at the interface of constitutive materials allows for jumps of the temperature field. The transmission conditions need to be handled carefully and efficiently. The main concerns are accuracy and feasibility. Hybrid dual formulations are recommended here as the most popular mixed finite elements are well adapted to account for the discontinuity of the temperature field. We therefore write the discretization of the heat problem by mixed finite elements and perform its numerical analysis. Of course, applying Lagrangian finite elements is possible in simple composite media but it turns out to be problematic for complex geometries. Nevertheless, we study the convergence of this finite element method to highlight some particularities related to the model under consideration and point out the effect of the contact resistance on the accuracy. Illustrative numerical experiments are finally provided to assess the theoretical findings.
\end{abstract}

Résumé: Nous considérons une équation qui modélise la diffusion de la température dans une mousse de graphite contenant des capsules de sel. Les conditions de transition de la température entre le graphite et le sel doivent être traitées correctement. Nous effectuons l'analyse de ce modèle et prouvons qu'il est bien posé. Puis nous en proposons une discrétisation par éléments finis et effectuons l'analyse a priori du problème discret. Quelques expériences numériques confirment l'intérêt de cette approche.

1 Sorbonne Universités, UTC, EA 2222, LMAC, 60205 Compiègne, France.

2 CNRS, UMR 7598, Laboratoire Jacques-Louis Lions, F-75005, Paris, France. Sorbonne Universités, UPMC Univ Paris 06, UMR 7598, LJLL, F-75005, Paris, France.

3 Université de Bordeaux, UB1-I2M UMR CNRS 5295, 33405 Talence, France.

4 ENIT-LAMSIN, BP 37, 1002 Tunis, Tunisia.

e-mail addresses: faker.ben-belgacem@utc.fr, bernardi@ann.jussieu.fr, faten.jelassi@lamsin.rnu.tn, maimouna.mintbrahim@u-bordeaux1.fr. 


\section{Introduction.}

Composite media are used in many technologies as a support for collecting heat energy of different origin for later needs. The principle consists in using a porous conductive medium saturated with material with high heat capacity. An example of these hybrid media is obtained by combining graphite foam with salt capsules. According to the size of the internal capsules or pores we speak of macro- or micro- encapsulated media. Most often, the heat conduction is significantly altered at the junction of different materials. The roughness of the interface creates gaps between materials, restricts the area with effective contact and contributes therefore to the imperfection of the heat flow. The lower thermal conductivity in those gaps ultimately causes a thermal resistance at the contact area. This phenomenon is called the thermal contact resistance. The fundamental effect observed in such a situation is the discontinuity of the temperature field across the interface. In most of the models engineers work with nowadays, the heat flux across the contact zone is proportional to the jump of the temperature. The proportionality constant is the thermal contact resistivity and its inverse is the thermal contact conductance (see [9], [20]).

To be more specific and express these ideas using mathematical language, let $\Omega$ be a connected bounded domain in $\mathbb{R}^{d}, d=2$ or 3 , with a Lipschitz-continuous boundary $\partial \Omega$. We consider a finite number of connected sub-domains $\omega_{i}$, called "capsules", so that each $\bar{\omega}_{i}$ is contained in $\Omega$, and the intersection of $\bar{\omega}_{i}$ and $\bar{\omega}_{j}$ for $i \neq j$ is empty. Next, we set:

$$
\Omega_{S}=\cup_{i} \omega_{i}, \quad \Omega_{G}=\Omega \backslash \bar{\Omega}_{S}, \quad \gamma=\partial \Omega_{S}=\cup_{i} \partial \omega_{i}
$$

The indices $S$ and $G$ call to mind salt and graphite. We consider also that the boundary $\partial \Omega$ is the disjoint union of two parts, $\Gamma_{D}$ and $\Gamma_{N}$. Both $\Gamma_{D}$ and $\Gamma_{N}$ are union of a finite number of connected components and $\Gamma_{D}$ has a positive $(d-1)$-measure. In this geometry, the differential system we intend to deal with is the steady heat transfer boundary value problem. The unknown, the temperature $T$ of the medium, satisfies therefore

$$
\begin{cases}-\operatorname{div}(\kappa \nabla T)=g & \text { in } \Omega_{S} \cup \Omega_{G}, \\ {\left[\kappa \partial_{n} T\right]=0} & \text { on } \gamma \\ R\left(\kappa \partial_{n}\left(\left.T\right|_{\Omega_{S}}\right)\right)=[T] & \text { on } \gamma \\ T=T_{D} & \text { on } \Gamma_{D} . \\ \kappa \partial_{n} T=0 & \text { on } \Gamma_{N} .\end{cases}
$$

Here $\boldsymbol{n}$ is the unit normal vector to $\partial \Omega$ exterior to $\Omega$ and also to $\gamma$ exterior to $\Omega_{S}$. The symbol [.] means the jump through $\gamma$, equal to the value on $\Omega_{G}$ minus the value on $\Omega_{S}$. Indeed, the temperature is likely discontinuous through $\gamma$. The parameter $R$ represents the thermal resistance at the interface $\gamma$ and $\kappa$ is the thermal conductivity. The heating data are the source $g$ and the external temperature $T_{D}$. 
Let us observe and emphasize on the fact that in real life the equation to consider is the transient heat transfer system (see [14], [19]). The point is that after using a timemarching scheme which is made without any particular technical obstacle, we are left with the quasi-static version of the problem to approximate in the space variable. This is the reason why we restrict this paper to the steady problem (1.2) which already contains all the specific difficulties users may encounter either in the theoretical or in the numerical grounds.

We consider here the finite element discretization of problem (1.2). The choice of the method to use is tightly dependent on the geometry of the composite medium. In simple configurations like the one depicted in Figure 1 (left part), users may apply the Lagrangian finite element method after introducing substantial modifications. The continuity is actually prescribed in each component $\Omega_{S}$ and $\Omega_{G}$ while jumps are allowed across the interfaces. The construction of the finite element space is therefore expected to account for this fact : local continuity/global discontinuity. The implementation should be made so to manage these two levels and may be troublesome. The difficulties can possibly be controlled by some domain decomposition procedures. Nonetheless, it turns out to be a pain in the neck in complicated geometry as is the case for densely composite media. We provide an illustration in the right part of Figure 1. Programming such a method and finding suitable solvers become tedious.
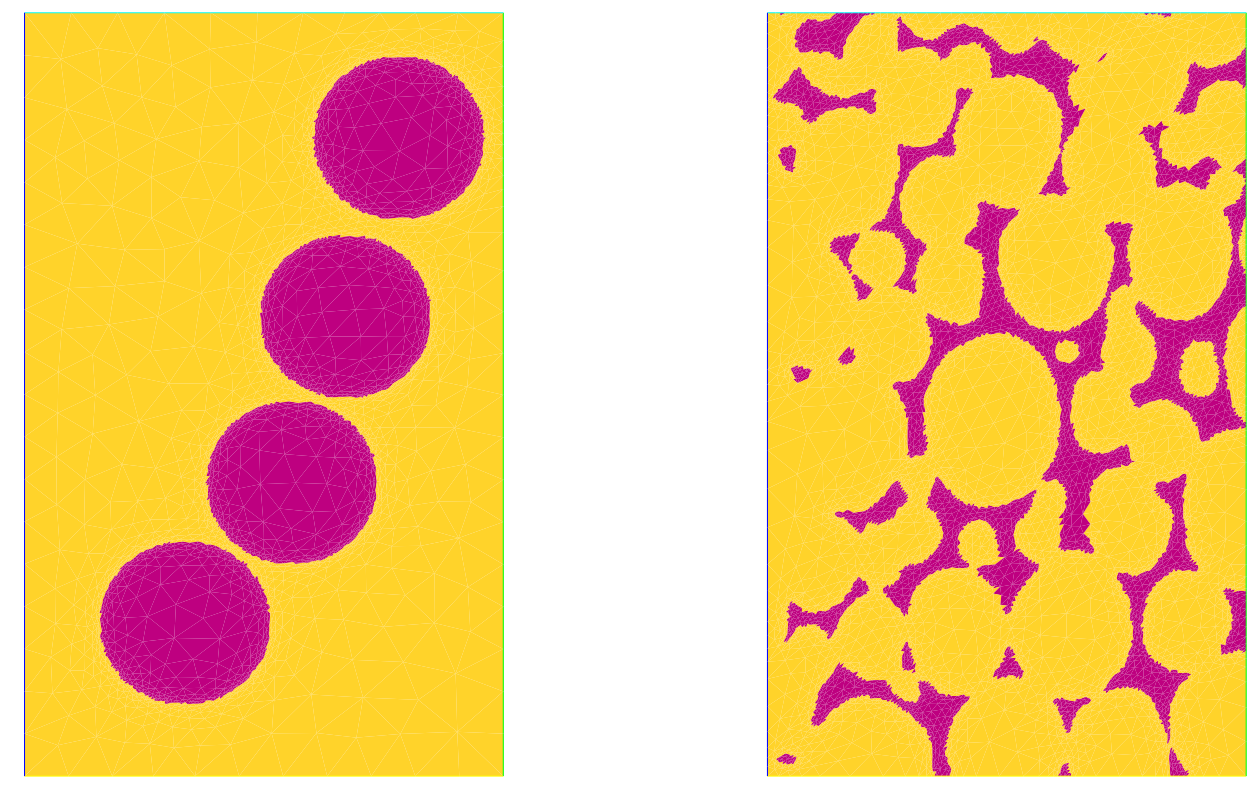

Figure 1 - Two examples of composite media.

An attractive alternative is offered by hybrid dual formulations as they bring about substantial advantages (see [17], [7]). The most important is that the construction of discrete spaces based on the mixed finite elements is natural and follows exactly the standard hybrid dual problem where the temperature field is continuous, i.e. $R=0$. Conditions of contact resistance at the interfaces are naturally accounted for in the mixed variational 
formulation. Hybrid Dual Finite Element Software can easily be enriched so to handle the problem we deal with here. The foundations of the finite elements library are not affected. Discontinuous Galerkin finite elements enjoy the similar properties as hybrid dual finite elements (see [2]). They can be used as well to solve problem (1.2). The cost is reduced to some suitable modifications to introduce on the variational problem. The structure of the finite elements should not be changed in softwares dedicated to elliptic problems.

The primary objective of this work is the description of both finite element methods and to conduct a numerical analysis for each of them. The outline of this article is as follows.

- In Section 2, we write the variational formulation of the problem and prove its wellposedness. The functional space fitting the problem is the broken Sobolev space. We consider also the hybrid dual variational model. The functional spaces currently used in the hybrid dual context allows jumps on the temperature field. They are not changed. We show how to take into account the interface condtions in the variational form. Before closing we conduct a brief discussion about the regularity of the solution and especially on the effect of the transmission conditions on the singularities born at the interfaces.

- Two finite element discretizations are proposed and analyzed for both variational problems in Sections 3 and 4, respectively. Using Aubin-Nitsche method together with a bootstrapping argument enables us to prove a local super-convergence result in the most interesting configurations.

- In Section 5, we present a few numerical experiments to assess the theoretical findings.

Acknowledgement: We are deeply grateful to Professor Vivette Girault for valuable discussion on the subject of the paper. 


\section{Variational formulations and well-posedness.}

Throughout, we use the full scale of Hilbertian Sobolev spaces $H^{s}(\Omega)$ for any real number $s$ (see [16, Chap. 1] and [1, Chap. 3] for all details on these spaces). In view of the analysis of problem (1.2), we introduce the variational space

$$
\mathbb{V}=\left\{v \in L^{2}(\Omega) ; \quad v_{G}=\left.v\right|_{\Omega_{G}} \in H^{1}\left(\Omega_{G}\right) \text { and } v_{S}=\left.v\right|_{\Omega_{S}} \in H^{1}\left(\Omega_{S}\right)\right\}
$$

It is naturally endowed with the broken norm

$$
\|v\|_{\mathbb{V}}=\left(\|v\|_{H^{1}\left(\Omega_{G}\right)}^{2}+\|v\|_{H^{1}\left(\Omega_{S}\right)}^{2}\right)^{1 / 2} .
$$

This norm determines a Hilbertian structure on $\mathbb{V}$. We will need the subspace

$$
\mathbb{V}_{0}=\left\{v \in \mathbb{V} ; v=0 \text { on } \Gamma_{D}\right\}
$$

It is easily seen that $\mathbb{V}_{0}$ is closed in $\mathbb{V}$, it is thus a Hilbert space. In the subsequent, the restrictions of any function $v$ in $\mathbb{V}$ to $\Omega_{G}$ and $\Omega_{S}$ are denoted by $v_{G}$ and $v_{S}$, respectively.

We assume that the conductivity $\kappa$ and the resistivity $R$ belong to $L^{\infty}(\Omega)$ and $L^{\infty}(\gamma)$ respectively and are bounded away from zero,

$$
\inf _{\boldsymbol{x} \in \Omega} \kappa(\boldsymbol{x})>0, \quad \inf _{\tau \in \gamma} R(\tau)>0 .
$$

We use sometimes the notation $\kappa_{S}$ and $\kappa_{G}$ for the restriction functions of $\kappa$ to $\Omega_{S}$ and $\Omega_{G}$, respectively. We also introduce the conductance $\alpha=R^{-1}$.

\subsection{Variational formulation.}

Multiplying the first equation in (1.2) by a smooth function $v$ on $\Omega_{S}$, and after integrating by parts, we obtain that

$$
\int_{\Omega_{S}} \kappa(\nabla T)(\boldsymbol{x}) \cdot(\nabla v)(\boldsymbol{x}) d \boldsymbol{x}-\int_{\gamma}\left(\kappa \partial_{n} T\right)(\tau) v(\tau) d \tau=\int_{\Omega_{S}} g(\boldsymbol{x}) v(\boldsymbol{x}) d \boldsymbol{x}
$$

The normal $\boldsymbol{n}$ is exterior to $\Omega_{S}$ and $\tau$ denotes the tangential variable on $\gamma$. Similarly, multiplying the same equation by a smooth function $v$ vanishing on $\Gamma_{D}$ and integrate on $\Omega_{G}$ results in

$$
\int_{\Omega_{G}} \kappa(\nabla T)(\boldsymbol{x}) \cdot(\nabla v)(\boldsymbol{x}) d \boldsymbol{x}+\int_{\gamma}\left(\kappa \partial_{n} T\right)(\tau) v(\tau) d \tau=\int_{\Omega_{G}} g(\boldsymbol{x}) v(\boldsymbol{x}) d \boldsymbol{x}
$$


For any smooth function $v$ in $\mathbb{V}_{0}$, we need to transform the integral term at the interface $\gamma$ as follows

$$
\begin{aligned}
\int_{\gamma}\left(-\kappa_{S}\left(\partial_{n} T_{S}\right)(\tau)\right. & \left.v_{S}(\tau)+\kappa_{G}\left(\partial_{n} T_{G}\right)(\tau) v_{G}(\tau)\right) d \tau \\
= & \int_{\gamma}\left[\kappa \partial_{n} T\right](\tau) v_{G}(\tau) d \tau+\int_{\gamma} \kappa_{S}\left(\partial_{n} T_{S}\right)(\tau)[v](\tau) d \tau
\end{aligned}
$$

Combining all this with the interface conditions (second and third equation in (1.2)), we derive that, for all function $v$ in $\mathbb{V}_{0}$

$$
\int_{\Omega_{S} \cup \Omega_{G}} \kappa(\nabla T)(\boldsymbol{x}) \cdot(\nabla v)(\boldsymbol{x}) d \boldsymbol{x}+\int_{\gamma} R^{-1}[T](\tau)[v](\tau) d \tau=\int_{\Omega_{S} \cup \Omega_{G}} g(\boldsymbol{x}) v(\boldsymbol{x}) d \boldsymbol{x} .
$$

As a consequence, we are led to consider the variational problem:

Find $T$ in $\mathbb{V}$ such that

$$
T=T_{D} \quad \text { on } \Gamma_{D},
$$

and

$$
\begin{aligned}
\forall v \in \mathbb{V}_{0}, \quad \int_{\Omega_{S} \cup \Omega_{G}} \kappa(\nabla T)(\boldsymbol{x}) \cdot(\nabla v)(\boldsymbol{x}) d \boldsymbol{x}+\int_{\gamma} \alpha[T](\tau)[v](\tau) d \tau \\
\quad=\int_{\Omega_{S} \cup \Omega_{G}} g(\boldsymbol{x}) v(\boldsymbol{x}) d \boldsymbol{x} .
\end{aligned}
$$

The proof of the next proposition easily follows from the previous lines and from the density of $\mathscr{D}\left(\bar{\Omega}_{S}\right) \times \mathscr{D}\left(\bar{\Omega}_{G}\right)$ into $\mathbb{V}$, however it requires a further assumption which is not restrictive (sufficient conditions for it are given in [3]).

Proposition 2.1. Assume that the partition of $\partial \Omega$ into $\Gamma_{D}$ and $\Gamma_{N}$ is sufficiently smooth for $\mathscr{D}\left(\bar{\Omega}_{G} \backslash \Gamma_{D}\right)$ to be dense in the space

$$
\left\{v \in H^{1}\left(\Omega_{G}\right) ; v=0 \text { on } \Gamma_{D}\right\} \text {. }
$$

Problems (1.2) and (2.3)-(2.4) are equivalent, in the sense that any function in $\mathbb{V}$ is a solution of (1.2) in the distribution sense if and only if it is a solution of (2.3)-(2.4).

Proof. Let $T$ be a solution of problem (2.3)-(2.4). By taking $v$ successively in $\mathscr{D}\left(\Omega_{G}\right)$, in $\mathscr{D}\left(\Omega_{S}\right)$, in $\mathscr{D}(\Omega)$ and finally in $\mathscr{D}\left(\Omega_{G} \cup \Omega_{S}\right)$ and using the same equations as previously, we derive that it is solution of (1.2) in the distribution sense. The converse property follows by the same arguments and by noting that the space

$$
\left\{v \in L^{2}(\Omega) ; v_{G} \in \mathscr{D}\left(\bar{\Omega}_{G} \backslash \Gamma_{D}\right) \text { and } v_{S} \in \mathscr{D}\left(\bar{\Omega}_{S}\right)\right\}
$$


is dense in $\mathbb{V}_{0}$.

Now, establishing the well-posedness of problem (2.3)-(2.4) requires to study the coerciveness of the bilinear form

$$
a(T, v)=\int_{\Omega_{S} \cup \Omega_{G}} \kappa(\nabla T)(\boldsymbol{x}) \cdot(\nabla v)(\boldsymbol{x}) d \boldsymbol{x}+\int_{\gamma} \alpha[T](\tau)[v](\tau) d \tau .
$$

Indeed, we have the following lemma.

Lemma 2.2. The mapping: $v \mapsto a(v, v)^{1 / 2}$ is a norm on $\mathbb{V}_{0}$ equivalent to the norm $\|v\|_{\mathbb{V}}$.

Proof. We first check out that $v \mapsto a(v, v)^{1 / 2}$ is a norm. Let then $v$ be a function in $\mathbb{V}_{0}$ such that $a(v, v)=0$. Thus, $\nabla v$ is zero on $\Omega_{G}$ and on $\Omega_{S}$ so that $v$ is equal to a constant $v_{G}$ on $\Omega_{G}$, to a constant $v_{S}$ on $\Omega_{S}$. It follows from the boundary condition on $\Gamma_{D}$ that $v_{G}$ is zero. Moreover, since the jump $[v]$ vanishes on $\gamma$ then $v_{S}$ is equal to $v_{G}$, hence to zero. All this yields that $v$ is zero.

Now, to show the equivalence with the norm $\|\cdot\|_{\mathbb{V}}$, observe that the continuity of the trace from $H^{1}\left(\Omega_{G}\right)$ and $H^{1}\left(\Omega_{S}\right)$ into $L^{2}(\gamma)$ yields that the norm $\|\cdot\|_{\mathbb{V}}$ is equivalent to the norm defined by

$$
v \mapsto a(v, v)^{1 / 2}+\left(\|v\|_{L^{2}\left(\Omega_{G}\right)}^{2}+\|v\|_{L^{2}\left(\Omega_{S}\right)}^{2}\right)^{1 / 2} .
$$

The kernel of the first term is reduced to zero and, due to the compact imbeddings of $H^{1}\left(\Omega_{G}\right)$ into $L^{2}\left(\Omega_{G}\right)$ and of $H^{1}\left(\Omega_{S}\right)$ into $L^{2}\left(\Omega_{S}\right)$, the second term is compact. The desired equivalence property is therefore a direct consequence of the Peetre-Tartar lemma, see [10, Chap. I, Thm 2.1].

The well-posedness result for the variational problem is a consequence of this lemma and Lax-Milgram theorem.

Corollary 2.3. For any data $g$ in $L^{2}(\Omega)$ and $T_{D}$ in $H^{1 / 2}\left(\Gamma_{D}\right)$, problem $(2.3)-(2.4)$ has a unique solution $T$ in $\mathbb{V}$. Moreover this solution satisfies

$$
\|T\|_{\mathbb{V}} \leq c\left(\|g\|_{L^{2}(\Omega)}+\left\|T_{D}\right\|_{H^{1 / 2}\left(\Gamma_{D}\right)}\right) .
$$

Proof. There exists a lifting $\bar{T}_{D}$ in $H^{1}\left(\Omega_{G}\right)$ of any extension of $T_{D}$ in $H^{1 / 2}(\partial \Omega)$ which vanishes on $\gamma$ and such that

$$
\left\|\bar{T}_{D}\right\|_{H^{1}\left(\Omega_{G}\right)} \leq c\left\|T_{D}\right\|_{H^{1 / 2}\left(\Gamma_{D}\right)} .
$$

Thus, writing the problem satisfied by $T_{0}=T-\bar{T}_{D}$ and noting that it belongs to $\mathbb{V}_{0}$, we deduce by combining the Lax-Milgram lemma with the ellipticity property proved in Lemma 2.2 that the problem has a unique solution. Moreover, the function $T=T_{0}+\bar{T}_{D}$ satisfies (2.7). 
In spite of the discontinuity of the temperature solution $T$, the maximum principle holds true.

Proposition 2.4. When $g$ is equal to zero, for any data $T_{D}$ in $H^{1 / 2}\left(\Gamma_{D}\right) \cap L^{\infty}\left(\Gamma_{D}\right)$, the solution $T$ of problem (2.3)-(2.4) satisfies for a.e. $\boldsymbol{x}$ in $\Omega$

$$
\min \left\{0, \inf _{\tau \in \Gamma_{D}} T_{D}(\tau)\right\} \leq T(\boldsymbol{x}) \leq \max \left\{0, \sup _{\tau \in \Gamma_{D}} T_{D}(\tau)\right\}
$$

Proof. Since the proofs of the two inequalities are fully identical, we only check out the second one. Set $M=\max \left\{0, \sup _{\tau \in \Gamma_{D}} T_{D}(\tau)\right\}$. Then, we observe that the function $T-M$ satisfies

$$
\forall v \in \mathbb{V}_{0}, \quad \int_{\Omega_{S} \cup \Omega_{G}} \kappa(\nabla(T-M))(\boldsymbol{x}) \cdot(\nabla v)(\boldsymbol{x}) d \boldsymbol{x}+\int_{\gamma} \alpha[T-M](\tau)[v](\tau) d \tau=0 .
$$

We choose $v$ equal to $(T-M)_{+}=\max \{T-M, 0\}$. Given that $T-M$ belongs to $\mathbb{V}$, then $(T-M)_{+}$lies also in $\mathbb{V}$. Moreover, based on the definition of $M$ this function belongs to $\mathbb{V}_{0}$. We have that

$$
\int_{\Omega_{S} \cup \Omega_{G}} \kappa\left(\nabla(T-M)_{+}\right)^{2} d \boldsymbol{x}+\int_{\gamma} \alpha[T-M](\tau)\left[(T-M)_{+}\right](\tau) d \tau=0
$$

Using the fact that $(r-s)\left(r_{+}-s_{+}\right) \geq 0$ for all real numbers $r, s$, we derive that

$$
\int_{\gamma} \alpha[(T-M)](\tau)\left[(T-M)_{+}\right](\tau) d \tau \geq 0
$$

As a result, both terms in the previous equation are zero. Thus, owing to Lemma 2.2, the function $(T-M)_{+}$vanishes identically, whence the desired result.

\subsection{Hybrid dual formulation.}

The hybrid dual functional framework adapted to our problem is easy to set and to cope with. It proceeds by the introduction of a second unknown, namely the quantity $\boldsymbol{p}=\kappa \nabla T$ (see [17], [7, Chap. I, §3 and §4]). The flux conservation across the interface prompts us to consider the space

$$
H(\operatorname{div} ; \Omega)=\left\{\boldsymbol{q} \in L^{2}(\Omega)^{d} ; \operatorname{div} \boldsymbol{q} \in L^{2}(\Omega)\right\}
$$

We recall from [10, Chap. I, Thm 2.5] that the trace operator: $\boldsymbol{q} \mapsto \boldsymbol{q} \cdot \boldsymbol{n}$ is continuous from $H(\operatorname{div} ; \Omega)$ onto the space $H^{-1 / 2}(\partial \Omega)$. From now on, we denote by $\langle\cdot, \cdot\rangle_{\partial \Omega}$ the duality pairing between $H^{-1 / 2}(\partial \Omega)$ and $H^{1 / 2}(\partial \Omega)$, and by $\langle\cdot, \cdot\rangle_{\Gamma_{D}}$ the duality pairing between the 
space $H^{1 / 2}\left(\Gamma_{D}\right)$ and its dual space. To step further and to fully take into account the interface conditions we need to work in the space

$$
\mathbb{X}=\left\{\boldsymbol{q} \in H(\operatorname{div} ; \Omega) ; \boldsymbol{q} \cdot \boldsymbol{n}=0 \text { on } \Gamma_{N} \text { and }\left.(\boldsymbol{q} \cdot \boldsymbol{n})\right|_{\gamma} \in L^{2}(\gamma)\right\}
$$

It is a Hilbert space when equipped with the norm

$$
\|\boldsymbol{q}\|_{\mathbb{X}}=\left(\|\boldsymbol{q}\|_{L^{2}(\Omega)^{d}}^{2}+\|\operatorname{div} \boldsymbol{q}\|_{L^{2}(\Omega)}^{2}+\|\boldsymbol{q} \cdot \boldsymbol{n}\|_{L^{2}(\gamma)}^{2}\right)^{1 / 2} .
$$

An alternative to the variational problem (2.3)-(2.4) consists in considering the following hybrid dual problem:

Find $(T, \boldsymbol{p})$ in $L^{2}(\Omega) \times \mathbb{X}$ such that

$$
\begin{aligned}
& \forall \boldsymbol{q} \in \mathbb{X}, \quad \int_{\Omega} \kappa^{-1} \boldsymbol{p}(\boldsymbol{x}) \cdot \boldsymbol{q}(\boldsymbol{x}) d \boldsymbol{x}+\int_{\gamma} R(\boldsymbol{p} \cdot \boldsymbol{n})(\tau)(\boldsymbol{q} \cdot \boldsymbol{n})(\tau) d \tau \\
&+\int_{\Omega}(\operatorname{div} \boldsymbol{q})(\boldsymbol{x}) T(\boldsymbol{x}) d \boldsymbol{x}=\left\langle T_{D}, \boldsymbol{q} \cdot \boldsymbol{n}\right\rangle_{\Gamma_{D}}, \\
& \forall v \in L^{2}(\Omega), \quad \int_{\Omega}(\operatorname{div} \boldsymbol{p})(\boldsymbol{x}) v(\boldsymbol{x}) d \boldsymbol{x}=-\int_{\Omega} g(\boldsymbol{x}) v(\boldsymbol{x}) d \boldsymbol{x} .
\end{aligned}
$$

This problem is of standard saddle point type, and it can be noted that no jump appears in its formulation. We start by proving the well-posedness of problem (2.11). The kernel $\mathbb{K}$ defined by

$$
\mathbb{K}=\left\{\boldsymbol{q} \in \mathbb{X} ; \forall v \in L^{2}(\Omega), \int_{\Omega}(\operatorname{div} \boldsymbol{q})(\boldsymbol{x}) v(\boldsymbol{x}) d \boldsymbol{x}=0\right\},
$$

is obviously characterized by

$$
\mathbb{K}=\{\boldsymbol{q} \in \mathbb{X} ; \operatorname{div} \boldsymbol{q}=0 \text { in } \Omega\}
$$

Thanks to the definition $(2.10)$ of the norm $\|\cdot\|_{\mathbb{X}}$, this yields the following result.

Lemma 2.5. The bilinear form:

$$
(\boldsymbol{p}, \boldsymbol{q}) \mapsto \int_{\Omega} \kappa^{-1} \boldsymbol{p}(\boldsymbol{x}) \cdot \boldsymbol{q}(\boldsymbol{x}) d \boldsymbol{x}+\int_{\gamma} R(\boldsymbol{p} \cdot \boldsymbol{n})(\tau)(\boldsymbol{q} \cdot \boldsymbol{n})(\tau) d \tau
$$

is elliptic on $\mathbb{K}$, with ellipticity constant equal to $\min \left\{\|\kappa\|_{L^{\infty}(\Omega)}^{-1}, \inf _{\tau \in \gamma} R(\tau)\right\}$.

The next step is to investigate the properties of the following mixed bilinear form:

$$
(\boldsymbol{q}, v) \mapsto \int_{\Omega}(\operatorname{div} \boldsymbol{q})(\boldsymbol{x}) v(\boldsymbol{x}) d \boldsymbol{x}
$$


Lemma 2.6. The following inf-sup condition holds for a positive constant $\beta$ only depending on $\Omega$

$$
\forall v \in L^{2}(\Omega), \quad \sup _{\boldsymbol{q} \in \mathbb{X}} \frac{\int_{\Omega}(\operatorname{div} \boldsymbol{q})(\boldsymbol{x}) v(\boldsymbol{x}) d \boldsymbol{x}}{\|\boldsymbol{q}\|_{\mathbb{X}}} \geq \beta\|v\|_{L^{2}(\Omega)} .
$$

Proof. It is processed as in [18] after adding some modifications. For any $v$ in $L^{2}(\Omega)$, we consider the solution $w$ of the Laplace equation

$$
\begin{cases}-\Delta w=-v & \text { in } \Omega \\ w=0 & \text { on } \Gamma_{D} \\ \partial_{n} w=0 & \text { on } \Gamma_{N}\end{cases}
$$

Obviously, this solution belongs to $H^{1}(\Omega)$. Then the function $\boldsymbol{q}=\nabla w$ satisfies $\operatorname{div} \boldsymbol{q}=v$ and

$$
\left(\|\boldsymbol{q}\|_{L^{2}(\Omega)^{d}}^{2}+\|\operatorname{div} \boldsymbol{q}\|_{L^{2}(\Omega)}^{2}\right)^{1 / 2} \leq c\|v\|_{L^{2}(\Omega)} .
$$

Moreover, $\boldsymbol{q} \cdot \boldsymbol{n}=\partial_{n} w$ vanishes on $\Gamma_{N}$. Owing to the elliptic regularity (see $[11$, Section 2.2.2]), $w$ belongs to $H^{2}(\mathcal{O})$ for any smooth domain $\mathcal{O}$ such that $\overline{\mathcal{O}} \subset \Omega$. Since $\gamma$ is strictly contained in $\Omega$, there exists a neighborhood $\overline{\mathcal{O}}$ of $\gamma$ in $\Omega$ such that

$$
\|\boldsymbol{q} \cdot \boldsymbol{n}\|_{L^{2}(\gamma)}=\left\|\partial_{n} w\right\|_{L^{2}(\gamma)} \leq\|w\|_{H^{2}(\mathcal{O})} \leq c\|v\|_{L^{2}(\Omega)}
$$

This concludes the proof.

The well-posedness of problem (2.11) is now a consequence of Lemmas 2.5 and 2.6, see [10, Chap. I, Thm 4.1] and [7, Chap. II, Thm 1.1] for instance.

Theorem 2.7. For any data $g$ in $L^{2}(\Omega)$ and $T_{D}$ in $H^{1 / 2}\left(\Gamma_{D}\right)$, problem (2.11) has a unique solution $(T, \boldsymbol{p})$ in $L^{2}(\Omega) \times \mathbb{X}$. Moreover this solution satisfies

$$
\|T\|_{L^{2}(\Omega)}+\|\boldsymbol{p}\|_{\mathbb{X}} \leq c\left(\|g\|_{L^{2}(\Omega)}+\left\|T_{D}\right\|_{H^{1 / 2}\left(\Gamma_{D}\right)}\right) .
$$

We conclude by comparing problem (2.11) with problem (1.2) (or equivalently with problem (2.3)-(2.4) thanks to Proposition 2.1).

Proposition 2.8. Assume that the partition of $\partial \Omega$ into $\Gamma_{D}$ and $\Gamma_{N}$ is sufficiently smooth for $\mathscr{D}\left(\Omega \cup \Gamma_{D}\right)$ to be dense in the space $\mathbb{X}$. Problems (1.2) and (2.11) are equivalent, in the sense that any function $T$ in $\mathbb{V}$ is a solution of (1.2) in the distribution sense if and only if the pair $(T, \boldsymbol{p}=\kappa \nabla T)$ is the solution of problem (2.11).

Proof. If $(T, \boldsymbol{p})$ stands for a solution of problem $(2.11)$, we consider this problem with $\boldsymbol{q}$ in $\mathscr{D}\left(\Omega_{G}\right)^{d} \cup \mathscr{D}\left(\Omega_{S}\right)^{d}$ and $v$ in $\mathscr{D}(\Omega)$. This yields

$$
\kappa^{-1} \boldsymbol{p}=\nabla T \quad \text { in } \Omega_{G} \cup \Omega_{S} \quad \text { and } \quad \operatorname{div} \boldsymbol{p}=-g \quad \text { in } \Omega,
$$


whence the equality $\boldsymbol{p}=\kappa \nabla T$ and also the first equation in (1.2). Since $\boldsymbol{p}$ belongs to $H(\operatorname{div} ; \Omega)$, this also implies the second equation in (1.2). Taking $\boldsymbol{q}$ in $\mathscr{D}(\Omega)$ and integrating by parts in (2.11) leads to the third equation in (1.2). The fourth equation is obtained by taking $\boldsymbol{q}$ in $\mathscr{D}\left(\Omega_{G} \cup \Gamma_{D}\right)^{d}$ and the fifth one follows from the fact that $\boldsymbol{p}$ belongs to $\mathbb{X}$. Thus, $T$ belongs to $\mathbb{V}$ and is a solution of (1.2). The converse property follows from the same arguments, together with the density assumption and the density of $\mathscr{D}(\Omega)$ into $L^{2}(\Omega)$.

\subsection{Regularity and singularities.}

The investigation of the accuracy of any finite element method applied to problem (1.2) requires to know the regularity of the solution $T$. It is admitted that the regularity of the solution is tightly connected to the smoothness of the data and of the geometry. Let us have a first look at the case where the geometry is smooth. To avoid the well known effect of mixed boundary conditions, we suppose that the Neumann portion is empty, that is $\Gamma_{N}=\emptyset$. We focus on the case where the resistivity $R$ is constant and the conductivity $\kappa$ is piecewise constant and takes two constant values $\kappa_{G}$ and $\kappa_{S}$ in $\Omega_{G}$ and $\Omega_{S}$ respectively. Similar results are valid for regular space varying $\kappa$ and $R$.

Proposition 2.9. Assume that the boundaries $\partial \Omega$ and $\gamma$ are of class $\mathscr{C}^{1,1}$. Then $T_{S}$ belongs to $H^{2}\left(\Omega_{S}\right)$. If in addition, the boundary datum $T_{D}$ belongs to $H^{3 / 2}(\partial \Omega)$, then $T_{G}$ also belongs to $H^{2}\left(\Omega_{G}\right)$.

Proof. Restricted to $\Omega_{S}$, the temperature $T$ is the solution of the Laplace equation with Neumann boundary condition

$$
\begin{cases}-\kappa_{S} \Delta T_{S}=g & \text { in } \Omega_{S}, \\ \kappa_{S} \partial_{n} T_{S}=\varphi & \text { on } \gamma,\end{cases}
$$

where the function $\varphi=\alpha[T]$ belongs to $H^{1 / 2}(\gamma)$. The desired result follows from [11, Thm $2.2 .2 .5]$.

Let us turn to the regularity on $\Omega_{G}$. The temperature $T$ is the solution of

$$
\begin{cases}-\kappa_{G} \Delta T_{G}=g & \text { in } \Omega_{G}, \\ \kappa_{G} \partial_{n} T_{G}=\psi & \text { on } \gamma, \\ T_{G}=T_{D} & \text { on } \Gamma_{D} .\end{cases}
$$

The function $\psi=\kappa_{S} \partial_{n} T_{S}$ is in $H^{1 / 2}(\gamma)$. Consequently, the solution $T_{G}$ belongs to $H^{2}\left(\Omega_{G}\right)$.

Remark 2.10. If the data and the geometry are highly smooth, a bootstrapping argument enables us to check that the local temperature fields $T_{G}$ and $T_{S}$ are also highly regular notwithstanding the discontinuity of the global temperature $T$. 
In the practice the geometry may not be so idealistic. We consider then more realistic cases where the geometry may have singular points or lines.

Proposition 2.11. Assume that any connected component $\omega_{i}$ of $\Omega_{S}$ is convex. Then the temperature $T_{S}$ belongs to $H^{2}\left(\Omega_{S}\right)$. Moreover, if the boundary datum $T_{D}$ belongs to $H^{3 / 2}(\partial \Omega)$, then the solution $T_{G}$ belongs to $H^{1+r}\left(\Omega_{G}\right)$, for some $r$ with $1 / 2 \leq r<1$. The following stability holds

$$
\left\|T_{S}\right\|_{H^{2}\left(\Omega_{S}\right)}+\left\|T_{G}\right\|_{H^{1+r}\left(\Omega_{G}\right)} \leq c\left(\|g\|_{L^{2}(\Omega)}+\left\|T_{D}\right\|_{H^{3 / 2}(\partial \Omega)}\right) .
$$

Proof. The proof is conducted exactly as for the previous proposition. The conclusion can be achieved following [11, Thm 3.2.1.3]. We refer to [15] for the case of a general Lipschitz boundary.

Remark 2.12. To have a deeper insight on this issue, let us have a close glance on the two-dimensional problem. We pay a particular attention to the case where the sub-domain $\Omega_{S}$ is polygonal. The limitation on the regularity of $T_{G}$ is caused by the angular vertices of $\gamma$ which create re-entrant corners for the domain $\Omega_{G}$. Now, consider the corner $\boldsymbol{m}$ of a sector $\mathcal{V}$ included in $\Omega_{G}$, with an aperture of the angle $\eta$ in $] \pi, 2 \pi[$ : The temperature field $T_{G}$, solution of sub-problem (2.16) is expected to contain a singularity of type

$$
S_{G}(\varrho, \theta)=\varrho^{\frac{\pi}{\eta}} \cos \left(\frac{\pi}{\eta} \theta\right) \varphi(\varrho)
$$

The polar coordinates $(\varrho, \theta)$ are used with origin $\boldsymbol{m}$ and $\varphi$ is a cut-off function around $\boldsymbol{m}$. Now, assume that $\mathcal{V}$ does not contain any other vertex. Then the singular function $S_{G}$ belongs to any Sobolev space $H^{s}(\mathcal{V})$ provided that $s<1+\frac{\pi}{\eta}$ and can not be in $H^{2}(\mathcal{V})$. The questions now are: is such a singularity compatible with the interfaces conditions? Why $S_{G}$ does not affect $T_{S}$ through the flux conservation $\left[\kappa \partial_{n} T\right]=0$ ? The answer is yes: $S_{G}$ is compatible with conditions along $\gamma$. The flux $\kappa \partial_{n} T_{G}=0$ along both edges of the sector $\mathcal{V}$. The temperature $T_{S}$ sees the singularity $S_{G}$ through the condition

$$
\kappa_{S} \partial_{n} T_{S}=\alpha[T], \quad \text { on } \gamma
$$

This does not prevent $\kappa \partial_{n} T_{S}$ from belonging to $H^{1 / 2}(\gamma)$. Things happen as if the consequence of releasing the strong continuity on the temperature $T$, by the introduction of a resistivity of contact, is the cancellation of any effect of the singularity $S_{G}$ on $T_{S}$. This will be addressed later on in the numerical section and a comparison will be conducted with the case of continuous temperature field for which the resistivity is zero, i.e. $R=0$. 


\section{A Lagrange finite element discretization.}

Lagrange finite elements may be applied for the approximation of the variational equation (2.4) in the case of macro-encapsulation. The sub-domain $\Omega_{S}$ is therefore the disjoint union of few connected components $\omega_{i}$ with reasonable boundaries. Handling local continuous/global discontinuous finite elements is therefore possible. At least, it can be managed without particular troubles in the numerical implementation grounds. Experienced numerical practitioners may even build local meshes in $\Omega_{S}$ and $\Omega_{G}$ that do not match at the interface and use mortaring devices to glue corresponding finite element discretizations (see [4]). In spite of the high interest of such a mortar issue, we do not consider it here for conciseness.

Assume hence that $\Omega$ is a polygon $(d=2)$ or a polyhedron $(d=3)$. We also suppose that $\Omega_{S}$ is a union of polygons or polyhedra, so that the boundary $\gamma$ is a polygonal curve or surface. Let $\left(\mathcal{T}_{h}\right)_{h}$ be a regular family of triangulations of $\Omega$ by triangles or tetrahedra, in the sense that, for each $h$ :

- The boundary $\gamma$ is the union of edges $(d=2)$ or faces $(d=3)$ of elements of $\mathcal{T}_{h}$.

- The union of all elements of $\mathcal{T}_{h}$ is equal to $\bar{\Omega}$.

- Each part $\Gamma_{D}$ or $\Gamma_{N}$ of the boundary $\partial \Omega$ is the union of edges $(d=2)$ or faces $(d=3)$ of elements of $\mathcal{T}_{h}$.

- The intersection of any two different elements of $\mathcal{T}_{h}$, if not empty, is a vertex or a whole edge or a whole face of both triangles or tetrahedra.

- The ratio of the diameter $h_{K}$ of any element $K$ of $\mathcal{T}_{h}$ to the diameter of its inscribed circle or sphere is smaller than a constant independent of $h$.

As usual, $h$ stands for the maximum of the diameters $h_{K}, K \in \mathcal{T}_{h}$. In all that follows, $c$, $c^{\prime}, \ldots$ are generic constants that can vary from line to line but are always independent of the parameter $h$.

Before defining the discrete space, let us introduce the local triangulations, for each mesh-size $h$,

$$
\mathcal{T}_{h}^{S}=\left\{K \in \mathcal{T}_{h} ; K \subset \bar{\Omega}_{S}\right\}, \quad \mathcal{T}_{h}^{G}=\left\{K \in \mathcal{T}_{h} ; K \subset \bar{\Omega}_{G}\right\}
$$

The discrete spaces are then constructed as follows

$$
\begin{aligned}
\mathbb{V}_{h}=\left\{v_{h} \in \mathbb{V} ; \forall K \in \mathcal{T}_{h}^{S},\left.v_{h}\right|_{K} \in \mathcal{P}_{1}(K) \text { and } \forall K \in \mathcal{T}_{h}^{G},\left.v_{h}\right|_{K} \in \mathcal{P}_{1}(K)\right\}, \\
\mathbb{V}_{0 h}=\mathbb{V}_{h} \cap \mathbb{V}_{0},
\end{aligned}
$$

where $\mathcal{P}_{1}(K)$ stands for the space of affine functions on $K$. Extension to high-order discretizations is obvious.

The discrete problem is obtained from problem (2.3)-(2.4) by the Galerkin method. Denoting by $i_{h}^{D}$ the Lagrange interpolation operator on $\Gamma_{D}$ with values in the space of traces of elements in $\mathbb{V}_{h}$ and assuming the continuity of $T_{D}$, it reads 
Find $T_{h}$ in $\mathbb{V}_{h}$ such that

$$
T_{h}=i_{h}^{D} T_{D} \quad \text { on } \Gamma_{D}
$$

and

$$
\begin{aligned}
\forall v_{h} \in \mathbb{V}_{0 h}, \quad \int_{\Omega_{S} \cup \Omega_{G}} \kappa\left(\nabla T_{h}\right)(\boldsymbol{x}) \cdot\left(\nabla v_{h}\right)(\boldsymbol{x}) d \boldsymbol{x} & +\int_{\gamma} \alpha\left[T_{h}\right](\tau)\left[v_{h}\right](\tau) d \tau \\
& =\int_{\Omega_{S} \cup \Omega_{G}} g(\boldsymbol{x}) v_{h}(\boldsymbol{x}) d \boldsymbol{x} .
\end{aligned}
$$

Since $\mathbb{V}_{0 h}$ is imbedded in $\mathbb{V}_{0}$, the ellipticity property established in Lemma 2.2, combined with the Lax-Milgram lemma, immediately yields the well-posedness result.

Proposition 3.1. For any data $g$ in $L^{2}(\Omega)$ and $T_{D}$ continuous on $\bar{\Gamma}_{D}$, problem $(3.1)-(3.2)$ has a unique solution $T_{h}$ in $\mathbb{V}_{h}$. Moreover this solution satisfies

$$
\left\|T_{h}\right\|_{\mathbb{V}} \leq c\left(\|g\|_{L^{2}(\Omega)}+\left\|i_{h}^{D} T_{D}\right\|_{H^{\frac{1}{2}\left(\Gamma_{D}\right)}}\right) .
$$

\subsection{Error analysis.}

On account of the coerciveness by Lemma 2.2, one derive straightforwardly the following version of Céa's lemma (see [8, Thm 2.4.1])

$$
\left\|T-T_{h}\right\|_{\mathbb{V}} \leq c \inf _{S_{h} \in \mathbb{V}_{h}^{D}}\left\|T-S_{h}\right\|_{\mathbb{V}}
$$

where $\mathbb{V}_{h}^{D}$ stand for the affine space of functions in $\mathbb{V}_{h}$ equal to $i_{h}^{D} T_{D}$ on $\Gamma_{D}$. As a consequence, choosing $S_{h}$ equal to the interpolate of $T$ (see [5, Chap. IX, Prop. 1.4]) yields the desired error estimate.

Theorem 3.2. Assume that the solution $T$ of problem (2.3)-(2.4) is such that

$$
T_{S} \in H^{s+1}\left(\Omega_{S}\right) ; \quad T_{G} \in \times H^{r+1}\left(\Omega_{G}\right),
$$

for real numbers $s, r$ with $0 \leq s, r \leq 1$. Then, the following a priori error estimate holds between this solution and the solution $T_{h}$ of problem (3.1)-(3.2)

$$
\left\|T-T_{h}\right\|_{\mathbb{V}} \leq c\left(h^{s}\left\|T_{S}\right\|_{H^{s+1}\left(\Omega_{S}\right)}+h^{r}\left\|T_{G}\right\|_{H^{r+1}\left(\Omega_{G}\right)}\right)
$$

Remark 3.3. Theorem 3.2 is worth some comments. Estimate (3.6) sounds artificial as it is. The effective form of the accuracy should be

$$
\left\|T-T_{h}\right\|_{\mathbb{V}} \leq c h^{\min \{s, r\}}\left(\left\|T_{S}\right\|_{H^{s+1}\left(\Omega_{S}\right)}+\left\|T_{G}\right\|_{H^{r+1}\left(\Omega_{G}\right)}\right) .
$$


Céa's lemma, at the basis of that error estimate, fails to uncouple what happens in $\Omega_{S}$ and in $\Omega_{G}$. In most of the real-life geometries the capsules $\omega_{i}$ are convex. According to Proposition 2.11, the temperature field $T$ enjoys more regularity within $\Omega_{S}$ than in $\Omega_{G}$. Indeed, we have $s=1$ and $r \in] 1 / 2,1$ [; thus $T_{S}$ belongs to $H^{2}\left(\Omega_{S}\right)$ but $T_{G}$ does not belong to $H^{2}\left(\Omega_{S}\right)$. Numericists and users may wonder whether a better accuracy within $\Omega_{S}$ may be derived.

\subsection{Super-convergence for realistic geometries.}

We pursue an improved accuracy in the internal sub-domain $\Omega_{S}$, when the components $\omega_{i}$ are convex. This is most often the case in the practice. The energy norm of the error decays actually like $h$ rather than $h^{r}$ with $r<1$, as predicted in Theorem 3.2.

We choose once again to develop the basic ideas in a simple context to avoid secondary technicalities that can be coped with following the specialized literature. We assume then that the conductivity is piecewise constant and takes the two constant values $\kappa_{S}$ in $\Omega_{S}$ and $\kappa_{G}$ in $\Omega_{G}$. We consider also that $\Gamma_{N}=\emptyset$ for simplicity. The methodology we develop consists in using the Aubin-Nitsche duality approach, then we call for a GagliardoNirenberg inequality to obtain enhanced convergence rate at the interfaces and finally extend the result to $\Omega_{S}$ by a bootstrapping argument.

Proposition 3.4. Assume $g$ in $L^{2}(\Omega)$ and $T_{D}$ in $H^{3 / 2}(\partial \Omega)$. Let all the connected components $\bar{\omega}_{i}$ of $\bar{\Omega}_{S}$ be convex. Then, the following a priori error estimate holds between the solution $T$ of problem (2.3)-(2.4) and the solution $T_{h}$ of problem (3.1)-(3.2)

$$
\left\|T-T_{h}\right\|_{L^{2}(\Omega)} \leq c h^{2 r}\left(\|g\|_{L^{2}(\Omega)}+\left\|T_{D}\right\|_{H^{3 / 2}(\partial \Omega)}\right) .
$$

The real number $r$ lies in $[1 / 2,1[$ and is defined in Proposition 2.11.

Proof. Observe first that by Theorem 3.2 and owing to the stability (2.17), we have

$$
\left\|T-T_{h}\right\|_{\mathbb{V}} \leq c h^{r}\left(\|g\|_{L^{2}(\Omega)}+\left\|T_{D}\right\|_{H^{3 / 2}(\partial \Omega)}\right) .
$$

Next, to proceed with Aubin-Nitsche duality we start from the formula

$$
\left\|T-T_{h}\right\|_{L^{2}(\Omega)}=\sup _{f \in L^{2}(\Omega)} \frac{\int_{\Omega_{S} \cup \Omega_{G}} f(\boldsymbol{x})\left(T-T_{h}\right)(\boldsymbol{x}) d \boldsymbol{x}}{\|f\|_{L^{2}(\Omega)}},
$$

and, for each $f$ in $L^{2}(\Omega)$, we solve the problem (we use here the notation (2.6) for brevity): Find $w$ in $\mathbb{V}_{0}$ such that

$$
\forall v \in \mathbb{V}_{0}, \quad a(v, w)=\int_{\Omega_{S} \cup \Omega_{G}} f(\boldsymbol{x}) v(\boldsymbol{x}) d \boldsymbol{x} .
$$


Thus, we have obviously

$$
\int_{\Omega_{S} \cup \Omega_{G}} f(\boldsymbol{x})\left(T-T_{h}\right)(\boldsymbol{x}) d \boldsymbol{x}=a\left(T-T_{h}, w\right),
$$

so that, by using problems $(2.3)-(2.4)$ and (3.1)-(3.2), for any $w_{h}$ in $\mathbb{V}_{0 h}$,

$$
\int_{\Omega_{S} \cup \Omega_{G}} f(\boldsymbol{x})\left(T-T_{h}\right)(\boldsymbol{x}) d \boldsymbol{x}=a\left(T-T_{h}, w-w_{h}\right) \leq c\left\|T-T_{h}\right\|_{\mathbb{V}}\left\|w-w_{h}\right\|_{\mathbb{V}}
$$

Calling for Proposition 2.11 yields that $w_{S}$ and $w_{G}$ belong to $H^{2}\left(\Omega_{S}\right)$ and $H^{1+r}\left(\Omega_{G}\right)$, and satisfy

$$
\left\|w_{S}\right\|_{H^{2}\left(\Omega_{S}\right)}+\left\|w_{G}\right\|_{H^{1+r}\left(\Omega_{G}\right)} \leq c\|f\|_{L^{2}(\Omega)} .
$$

Choosing $w_{h}$ equal to the Lagrange interpolate of $w$ yields

$$
\left\|w-w_{h}\right\|_{\mathbb{V}} \leq c h^{r}\|f\|_{L^{2}(\Omega)}
$$

Combining all this with estimate (3.8) provides the desired result and ends the proof.

Now, the second step consists in deriving an error estimate on the $L^{2}$-norm of the jump of $\left[T-T_{h}\right]$ along the interface $\gamma$.

Lemma 3.5. If assumptions of Proposition 3.4 are valid then the following estimate holds

$$
\left\|\left[T-T_{h}\right]\right\|_{L^{2}(\gamma)} \leq c h^{\frac{3}{2} r}\left(\|g\|_{L^{2}(\Omega)}+\left\|T_{D}\right\|_{H^{3 / 2}(\partial \Omega)}\right) .
$$

Proof. It follows from the trace theorem, see [6, Thm 1.6.6] for instance, that, for all $v$ in $\mathbb{V}$,

$$
\|v\|_{L^{2}(\gamma)} \leq c\|v\|_{L^{2}\left(\Omega_{S}\right)}^{\frac{1}{2}}\|v\|_{H^{1}\left(\Omega_{S}\right)}^{\frac{1}{2}} .
$$

Of course, the same inequality holds with $\Omega_{S}$ replaced by $\Omega_{G}$. Applying this inequality to $T-T_{h}$ yields

$$
\left\|\left[T-T_{h}\right]\right\|_{L^{2}(\gamma)} \leq c\left\|T-T_{h}\right\|_{L^{2}(\Omega)}^{\frac{1}{2}}\left\|T-T_{h}\right\|_{\mathbb{V}}^{\frac{1}{2}}
$$

Using estimates (3.7) and (3.8) yields that

$$
\left\|\left[T-T_{h}\right]\right\|_{L^{2}(\gamma)} \leq c h^{\frac{3}{2} r}\left(\|g\|_{L^{2}(\Omega)}+\left\|T_{D}\right\|_{H^{3 / 2}(\partial \Omega)}\right)
$$

which is the desired result.

Remark 3.6. The same arguments as in the previous proof, see (3.10), also yield

$$
\left\|T_{S}-\left.T_{h}\right|_{\Omega_{S}}\right\|_{L^{2}(\gamma)} \leq c h^{\frac{3}{2} r}\left(\|g\|_{L^{2}(\Omega)}+\left\|T_{D}\right\|_{H^{3 / 2}(\partial \Omega)}\right) .
$$


Finally, we are in position to improve the error estimate in the sub-domain $\Omega_{S}$.

Theorem 3.7. If assumptions of Proposition 3.4 are valid then, the following a priori error estimate holds between the solution $T$ of problem (2.3)-(2.4) and the solution $T_{h}$ of problem $(3.1)-(3.2)$

$$
\left\|T-T_{h}\right\|_{H^{1}\left(\Omega_{S}\right)} \leq c h^{\min \left\{1, \frac{3}{2} r\right\}}\left(\|g\|_{L^{2}(\Omega)}+\left\|T_{D}\right\|_{H^{3 / 2}(\partial \Omega)}\right),
$$

for the parameter $r$ defined in Proposition 2.11.

Proof. Introduce first the notation $\mathbb{V}_{h, S}=\left.\mathbb{V}_{h}\right|_{\Omega_{S}}$. We start from

$$
\forall v_{h} \in \mathbb{V}_{h}, \quad a\left(T-T_{h}, v_{h}\right)=0 .
$$

Choosing $v_{h}$ such that $\left.v_{h}\right|_{\Omega_{G}}=0$ and setting $w_{h}=\left.v_{h}\right|_{\Omega_{S}} \in \mathbb{V}_{h, S}$ we obtain

$$
\forall w_{h} \in \mathbb{V}_{h, S}, \quad \int_{\Omega_{S}} \kappa\left(\nabla\left(T-T_{h}\right)\right)(\boldsymbol{x}) \cdot\left(\nabla w_{h}\right)(\boldsymbol{x}) d \boldsymbol{x}+\int_{\gamma} \alpha\left[T-T_{h}\right](\tau) w_{h}(\tau) d \tau=0
$$

Applying this equation yields, for any $S_{h}$ in $\mathbb{V}_{h, S}$,

$$
\begin{array}{r}
\int_{\Omega_{S}} \kappa\left(\nabla\left(T-T_{h}\right)\right)^{2}(\boldsymbol{x}), d \boldsymbol{x}=\int_{\Omega_{S}} \kappa\left(\nabla\left(T-T_{h}\right)\right)(\boldsymbol{x}) \cdot\left(\nabla\left(T-S_{h}\right)\right)(\boldsymbol{x}) d \boldsymbol{x} \\
-\int_{\gamma} \alpha\left[T-T_{h}\right](\tau)\left(S_{h}-T_{h}\right)(\tau) d \tau
\end{array}
$$

whence, by using triangle inequalities and the trace theorem in the last term,

$\left\|T-T_{h}\right\|_{H^{1}\left(\Omega_{S}\right)}^{2} \leq c\left\|T-S_{h}\right\|_{H^{1}\left(\Omega_{S}\right)}^{2}+c^{\prime}\left\|\left[T-T_{h}\right]\right\|_{L^{2}(\gamma)}\left(\left\|T-T_{h}\right\|_{L^{2}(\gamma)}+\left\|T-S_{h}\right\|_{L^{2}(\gamma)}\right)$.

Observing that $T_{S}$ belongs to $H^{2}\left(\Omega_{S}\right)$, see Proposition 2.11, applying Lemma 3.5 and Remark 3.6 and using the error estimate of the general theory of finite elements, we derive for appropriate constants $c$ and $c^{\prime}$ depending on the data

which is the desired result.

$$
\left\|T-T_{h}\right\|_{H^{1}\left(\Omega_{S}\right)}^{2} \leq c h^{2}+c^{\prime} h^{3 r},
$$

Remark 3.8. According to estimate (3.12) the optimal accuracy in the sub-domain $\Omega_{S}$ is guaranteed when $r>\frac{2}{3}$. The convergence speed is then of order $\mathcal{O}(h)$. The previous argument can be iterated to improve the result. Unfortunately, this bootstrap technique does not lead to the full optimal estimate for $r$ close to $1 / 2$. So far the optimality is missing when the capsules $\omega_{i}$ have acute angles $(\leq \pi / 3)$. Notice that the convergence rate of the error with respect to the energy norm in $\Omega_{S}$ is anyway better than $\mathcal{O}\left(h^{5 / 6}\right)$. However, our feeling is that the optimality would be valid for any $r>1 / 2$. The numerical discussion realized later on confirms this claim.

Remark 3.9. Although, we expose the procedure for enhancing the convergence rate in a particular geometry. It can be extended to a larger class of configurations provided that the convergence rate the global error with respect to the $L^{2}$-norm is higher than the rate with respect to the $H^{1}$-norm. Furthermore, the arguments exposed here may be applied locally as well. One may focus on a particular convex component $\omega_{i}$ instead of the whole $\Omega_{S}$. 


\section{Hybrid dual Raviart-Thomas finite elements.}

For many interesting dense hybrid media as the one depicted in Figure 1, using Lagrange finite elements for the discretization of problem (1.2) compels practitioners to transform the finite elements Libraries which are the very foundation of most of existing Finite Element Computing Softwares. Users are reluctant to dive so deep in the programing layers (of the softwares). They prefer to work at the layers perceived as external layers. It is most often the level of the variational formulation writing.

With this respect, for the family of triangulations $\left(\mathcal{T}_{h}\right)_{h}$ introduced in Section 3 , the finite element framework needed for the hybrid dual formulation is exactly the same as the one used for continuous temperature field. Indeed, the discrete space of temperatures is defined to be

$$
\mathbb{M}_{h}=\left\{v_{h} \in L^{2}(\Omega) ; \forall K \in \mathcal{T}_{h},\left.v_{h}\right|_{K} \in \mathcal{P}_{0}(K)\right\},
$$

where $\mathcal{P}_{0}(K)$ stands for the space of constant functions on $K$. For the space aimed to approximate $\boldsymbol{p}$, we have decided to use the Raviart-Thomas element, introduced in [17], since it is well-known to be $H(\operatorname{div} ; \Omega)$-conforming. So we take

$$
\mathbb{X}_{h}=\left\{\boldsymbol{q}_{h} \in H(\operatorname{div} ; \Omega) ; \forall K \in \mathcal{T}_{h},\left.\boldsymbol{q}_{h}\right|_{K} \in R T_{0}(K)\right\}, \quad \mathbb{X}_{h}^{0}=\mathbb{X}_{h} \cap \mathbb{X}
$$

where $R T_{0}(K)$ is the space of restrictions to $K$ of polynomials of the form $\boldsymbol{a}+b \boldsymbol{x}, \boldsymbol{a} \in \mathbb{R}^{d}$, $b \in \mathbb{R}$.

Remark 4.1. It is of course possible to use higher order elements. We work with the low cost finite element spaces defined above only for simplicity. It is also possible to use the so-called $B D M_{1}$ space (due to Brezzi, Douglas and Marini) instead of $R T_{0}$. Many extensions are possible and our feeling is that they do not arise any specific difficulty.

The discrete problem is now constructed from problem (2.11) by the Galerkin method, it reads

Find $\left(T_{h}, \boldsymbol{p}_{h}\right)$ in $\mathbb{M}_{h} \times \mathbb{X}_{h}^{0}$ such that

$$
\begin{array}{r}
\forall \boldsymbol{q}_{h} \in \mathbb{X}_{h}^{0}, \quad \int_{\Omega} \kappa^{-1} \boldsymbol{p}_{h}(\boldsymbol{x}) \cdot \boldsymbol{q}_{h}(\boldsymbol{x}) d \boldsymbol{x}+\int_{\gamma} R\left(\boldsymbol{p}_{h} \cdot \boldsymbol{n}\right)(\tau)\left(\boldsymbol{q}_{h} \cdot \boldsymbol{n}\right)(\tau) d \tau \\
+\int_{\Omega}\left(\operatorname{div} \boldsymbol{q}_{h}\right)(\boldsymbol{x}) T_{h}(\boldsymbol{x}) d \boldsymbol{x}=\left\langle T_{D}, \boldsymbol{q}_{h} \cdot \boldsymbol{n}\right\rangle_{\Gamma_{D}}, \\
\forall v_{h} \in \mathbb{M}_{h}, \quad \int_{\Omega}\left(\operatorname{div} \boldsymbol{p}_{h}\right)(\boldsymbol{x}) v_{h}(\boldsymbol{x}) d \boldsymbol{x}=-\int_{\Omega} g(\boldsymbol{x}) v_{h}(\boldsymbol{x}) d \boldsymbol{x} .
\end{array}
$$

Note that, due to the choice of $\mathbb{M}_{h}$ and $\mathbb{X}_{h}^{0}$, the discretization is fully conforming.

Proving the well-posedness of problem (4.3) relies on very similar arguments as for the continuous case. Let us introduce the kernel

$$
\mathbb{K}_{h}=\left\{\boldsymbol{q}_{h} \in \mathbb{X}_{h}^{0} ; \forall v_{h} \in \mathbb{M}_{h}, \int_{\Omega}\left(\operatorname{div} \boldsymbol{q}_{h}\right)(\boldsymbol{x}) v_{h}(\boldsymbol{x}) d \boldsymbol{x}=0\right\}
$$


Since, for each $\boldsymbol{q}_{h}$ in $\mathbb{X}_{h}^{0}$, the function $\operatorname{div} \boldsymbol{q}_{h}$ is constant on each element $K$ of $\mathcal{T}_{h}$, taking $v_{h}$ equal to $\operatorname{div} \boldsymbol{q}_{h}$ yields the next result.

Lemma 4.2. The kernel $\mathbb{K}_{h}$ is imbedded in the kernel $\mathbb{K}$.

Thus, the ellipticity property stated in Lemma 2.5 is still valid on $\mathbb{K}_{h}$. To proceed further, we introduce the so called Raviart-Thomas operator: if $\mathcal{E}_{h}$ stands for the set of all edges $(d=2)$ or faces $(d=3)$ of elements of $\mathcal{T}_{h}$, for any $\boldsymbol{q}$ in $H(\operatorname{div}, \Omega), \Pi_{h} \boldsymbol{q}$ belongs to $\mathbb{X}_{h}$ and satisfies

$$
\forall e \in \mathcal{E}_{h}, \quad \int_{e}\left(\Pi_{h} \boldsymbol{q} \cdot \boldsymbol{n}\right)(\tau) d \tau=\int_{e}(\boldsymbol{q} \cdot \boldsymbol{n})(\tau) d \tau
$$

It follows from the properties of the Raviart-Thomas element that the equations in (4.5) define $\Pi_{h}$ in a unique way, see [5, Chap. VII, Lemma 3.19] for instance. We now state some properties of this operator.

Lemma 4.3. The operator $\Pi_{h}$ is continuous from $\mathbb{X}$ into $\mathbb{X}_{h}^{0}$. Moreover it satisfies

$$
\forall \boldsymbol{q} \in \mathbb{X}, \quad\left\|\Pi_{h} \boldsymbol{q}\right\|_{\mathbb{X}} \leq c\|\boldsymbol{q}\|_{\mathbb{X}}
$$

Proof. It follows from the definition (4.5) of the operator $\Pi_{h}$ that it preserves the nullity of the normal component on $\Gamma_{N}$, hence maps $\mathbb{X}$ into $\mathbb{X}_{h}^{0}$. On the other hand, with each $e$ in $\mathcal{E}_{h}$, we associate the function $\varphi_{e}$ defined on each $K$ that contains $e$ by

$$
\boldsymbol{\varphi}_{e}(\boldsymbol{x})=\frac{\boldsymbol{x}-\boldsymbol{a}}{d \operatorname{meas}(K)}
$$

where $\boldsymbol{a}$ is the vertex of $K$ opposite to $e$, and equal to zero elsewhere. It is readily checked that

$$
\Pi_{h} \boldsymbol{q}=\sum_{e \in \mathcal{E}_{h}}\left(\int_{e}(\boldsymbol{q} \cdot \boldsymbol{n})(\tau) d \tau\right) \boldsymbol{\varphi}_{e},
$$

We now proceed in three steps.

1) It follow from the previous formula that

$$
\left\|\Pi_{h} \boldsymbol{q}\right\|_{L^{2}(\Omega)} \leq C\left(\|\boldsymbol{q}\|_{L^{2}(\Omega)}+\|\operatorname{div} \boldsymbol{q}\|_{L^{2}(\Omega)}\right) .
$$

2) Since the divergence of each $\varphi_{e}$ on any $K$ that contains $e$ is equal to $\frac{1}{\operatorname{meas}(K)}$, we have

$$
\int_{K}\left(\operatorname{div} \Pi_{h} \boldsymbol{q}\right)(\boldsymbol{x}) d \boldsymbol{x}=\int_{\partial K}(\boldsymbol{q} \cdot \boldsymbol{n})(\tau) d \tau=\int_{K}(\operatorname{div} \boldsymbol{q})(\boldsymbol{x}) d \boldsymbol{x}
$$

This leads to

$$
\left\|\operatorname{div} \Pi_{h} \boldsymbol{q}\right\|_{L^{2}(\Omega)} \leq\|\operatorname{div} \boldsymbol{q}\|_{L^{2}(\Omega)} .
$$


3) On the other hand, it is readily checked that, for each $e$ contained in $\gamma$, there holds

$$
\int_{e}\left(\Pi_{h} \boldsymbol{q} \cdot \boldsymbol{n}\right)(\tau) d \tau=\int_{e}(\boldsymbol{q} \cdot \boldsymbol{n})(\tau) d \tau
$$

Hence, we obtain

$$
\left\|\Pi_{h} \boldsymbol{q} \cdot \boldsymbol{n}\right\|_{L^{2}(\gamma)} \leq\|\boldsymbol{q} \cdot \boldsymbol{n}\|_{L^{2}(\gamma)}
$$

The desired result follows from (4.7), (4.9) and (4.10).

Fortunately, this technical proof leads to the inf-sup condition on the second form in problem (4.3).

Lemma 4.4. The following inf-sup condition holds for a positive constant $\beta_{*}$ independent of $h$

$$
\forall v_{h} \in \mathbb{M}_{h}, \quad \sup _{\boldsymbol{q}_{h} \in \mathbb{X}_{h}^{0}} \frac{\int_{\Omega}\left(\operatorname{div} \boldsymbol{q}_{h}\right)(\boldsymbol{x}) v_{h}(\boldsymbol{x}) d \boldsymbol{x}}{\left\|\boldsymbol{q}_{h}\right\|_{\mathbb{X}}} \geq \beta_{*}\left\|v_{h}\right\|_{L^{2}(\Omega)} .
$$

Proof. Let $v_{h}$ be any function in $\mathbb{M}_{h}$. In the proof of Lemma 2.6, we have exhibited a function $\boldsymbol{q}$ in $\mathbb{X}$ such that $\operatorname{div} \boldsymbol{q}=v_{h}$ and

$$
\|\boldsymbol{q}\|_{\mathbb{X}} \leq c\left\|v_{h}\right\|_{L^{2}(\Omega)}
$$

We have

$$
\int_{\Omega}\left(\operatorname{div} \Pi_{h} \boldsymbol{q}\right)(\boldsymbol{x}) v_{h}(\boldsymbol{x}) d \boldsymbol{x}=\left.\sum_{K \in \mathcal{T}_{h}} v_{h}\right|_{K} \int_{K}\left(\operatorname{div} \Pi_{h} \boldsymbol{q}\right)(\boldsymbol{x}) d \boldsymbol{x},
$$

whence from (4.8)

$$
\int_{\Omega}\left(\operatorname{div} \Pi_{h} \boldsymbol{q}\right)(\boldsymbol{x}) v_{h}(\boldsymbol{x}) d \boldsymbol{x}=\left.\sum_{K \in \mathcal{T}_{h}} v_{h}\right|_{K} \int_{K}(\operatorname{div} \boldsymbol{q})(\boldsymbol{x}) d \boldsymbol{x}=\left\|v_{h}\right\|_{L^{2}(\Omega)}^{2} .
$$

On the other hand, it follows from Lemma 4.3 that

$$
\left\|\Pi_{h} \boldsymbol{q}\right\|_{\mathbb{X}} \leq c\|\boldsymbol{q}\|_{\mathbb{X}} \leq c^{\prime}\left\|v_{h}\right\|_{L^{2}(\Omega)} .
$$

All this yields the desired condition.

The well-posedness of the discrete problem (4.3) is now a direct consequence of Lemmas 2.5 (combined with Lemma 4.2) and 4.4, see once more [10, Chap. I, Thm 4.1] or [7, Chap. II, Thm 1.1].

Theorem 4.5. For any data $g$ in $L^{2}(\Omega)$ and $T_{D}$ in $H^{\frac{1}{2}}\left(\Gamma_{D}\right)$, problem (4.3) has a unique solution $\left(T_{h}, \boldsymbol{p}_{h}\right)$ in $\mathbb{M}_{h} \times \mathbb{X}_{h}^{0}$. Moreover this solution satisfies

$$
\left\|T_{h}\right\|_{L^{2}(\Omega)}+\left\|\boldsymbol{p}_{h}\right\|_{\mathbb{X}} \leq c\left(\|g\|_{L^{2}(\Omega)}+\left\|T_{D}\right\|_{H^{\frac{1}{2}}\left(\Gamma_{D}\right)}\right) .
$$


We are also in a position to derive an a priori error estimate between the continuous and discrete solutions. We begin with the following version of the Strang's lemma.

Lemma 4.6. The following bound holds between the solution $(T, \boldsymbol{p})$ of problem $(2.11)$ and the solution $\left(T_{h}, p_{h}\right)$ of problem (4.3)

$$
\begin{aligned}
& \left\|\boldsymbol{p}-\boldsymbol{p}_{h}\right\|_{\mathbb{X}} \leq c\left\|\boldsymbol{p}-\Pi_{h} \boldsymbol{p}\right\|_{\mathbb{X}}, \\
& \qquad T-T_{h} \|_{L^{2}(\Omega)} \leq c\left(\left\|\boldsymbol{p}-\Pi_{h} \boldsymbol{p}\right\|_{\mathbb{X}}+\inf _{S_{h} \in \mathbb{M}_{h}}\left\|T-S_{h}\right\|_{L^{2}(\Omega)}\right) .
\end{aligned}
$$

Proof. We prove successively the two estimates.

1) We first observe from (4.8) that $\Pi_{h} \boldsymbol{p}-\boldsymbol{p}_{h}$ belongs to the kernel $\mathbb{K}_{h}$. Then, we derive from Lemmas 2.5 and 4.2 that

$$
\left\|\Pi_{h} \boldsymbol{p}-\boldsymbol{p}_{h}\right\|_{\mathbb{X}}^{2} \leq c\left(\int_{\Omega} \kappa^{-1}\left(\left(\Pi_{h} \boldsymbol{p}-\boldsymbol{p}_{h}\right)^{2}(\boldsymbol{x}) d \boldsymbol{x}+\int_{\gamma} R\left(\left(\Pi_{h} \boldsymbol{p}-\boldsymbol{p}_{h}\right) \cdot \boldsymbol{n}\right)^{2}\right)(\tau) d \tau\right) .
$$

By using first problem (4.3) and second problem (2.11), this yields

$$
\begin{aligned}
\left\|\Pi_{h} \boldsymbol{p}-\boldsymbol{p}_{h}\right\|_{\mathbb{X}}^{2} \leq c\left(\int_{\Omega} \kappa^{-1} \Pi_{h} \boldsymbol{p}(\boldsymbol{x}) \cdot\left(\Pi_{h} \boldsymbol{p}-\boldsymbol{p}_{h}\right)(\boldsymbol{x}) d \boldsymbol{x}\right. \\
\left.\quad+\int_{\gamma} R\left(\Pi_{h} \boldsymbol{p} \cdot \boldsymbol{n}\right)(\tau)\left(\left(\Pi_{h} \boldsymbol{p}-\boldsymbol{p}_{h}\right) \cdot \boldsymbol{n}\right)(\tau) d \tau-\left\langle T_{D}, \Pi_{h} \boldsymbol{p}-\boldsymbol{p}_{h}\right\rangle_{\Gamma_{D}}\right) \\
\leq c\left(\int_{\Omega} \kappa^{-1}\left(\Pi_{h} \boldsymbol{p}-\boldsymbol{p}\right)(\boldsymbol{x}) \cdot\left(\Pi_{h} \boldsymbol{p}-\boldsymbol{p}_{h}\right)(\boldsymbol{x}) d \boldsymbol{x}\right. \\
\left.\quad+\int_{\gamma} R\left(\left(\Pi_{h} \boldsymbol{p}-\boldsymbol{p}\right) \cdot \boldsymbol{n}\right)(\tau)\left(\left(\Pi_{h} \boldsymbol{p}-\boldsymbol{p}_{h}\right) \cdot \boldsymbol{n}\right)(\tau) d \tau\right) .
\end{aligned}
$$

A Cauchy-Schwarz inequality leads to

$$
\left\|\Pi_{h} \boldsymbol{p}-\boldsymbol{p}_{h}\right\|_{\mathbb{X}} \leq c\left\|\Pi_{h} \boldsymbol{p}-\boldsymbol{p}\right\|_{\mathbb{X}}
$$

and we conclude the bound for $\left\|\boldsymbol{p}-\boldsymbol{p}_{h}\right\|_{\mathbb{X}}$ thanks to a triangle inequality.

2) To prove the second estimate, we apply the inf-sup condition (4.11): For any $S_{h}$ in $\mathbb{M}_{h}$,

$$
\left\|T_{h}-S_{h}\right\|_{L^{2}(\Omega)} \leq \beta_{*}^{-1} \sup _{\boldsymbol{q}_{h} \in \mathbb{X}_{h}^{0}} \frac{\int_{\Omega}\left(\operatorname{div} \boldsymbol{q}_{h}\right)(\boldsymbol{x})\left(T_{h}-S_{h}\right)(\boldsymbol{x})(\boldsymbol{x}) d \boldsymbol{x}}{\left\|\boldsymbol{q}_{h}\right\|_{\mathbb{X}}} .
$$

By using problems (4.3) and (2.11), we obtain

$$
\begin{aligned}
& \int_{\Omega}\left(\operatorname{div} \boldsymbol{q}_{h}\right)(\boldsymbol{x})\left(T_{h}-S_{h}\right)(\boldsymbol{x})(\boldsymbol{x}) d \boldsymbol{x} \\
& =\left\langle T_{D}, \boldsymbol{q}_{h} \cdot \boldsymbol{n}\right\rangle_{\Gamma_{D}}-\int_{\Omega} \kappa^{-1} \boldsymbol{p}_{h}(\boldsymbol{x}) \cdot \boldsymbol{q}_{h}(\boldsymbol{x}) d \boldsymbol{x} \\
& \quad-\int_{\gamma} R\left(\boldsymbol{p}_{h} \cdot \boldsymbol{n}\right)(\tau)\left(\boldsymbol{q}_{h} \cdot \boldsymbol{n}\right)(\tau) d \tau-\int_{\Omega}\left(\operatorname{div} \boldsymbol{q}_{h}\right)(\boldsymbol{x}) S_{h}(\boldsymbol{x})(\boldsymbol{x}) d \boldsymbol{x} \\
& =\int_{\Omega} \kappa^{-1}\left(\boldsymbol{p}-\boldsymbol{p}_{h}\right)(\boldsymbol{x}) \cdot \boldsymbol{q}_{h}(\boldsymbol{x}) d \boldsymbol{x} \\
& \quad+\int_{\gamma} R\left(\left(\boldsymbol{p}-\boldsymbol{p}_{h}\right) \cdot \boldsymbol{n}\right)(\tau)\left(\boldsymbol{q}_{h} \cdot \boldsymbol{n}\right)(\tau) d \tau+\int_{\Omega}\left(\operatorname{div} \boldsymbol{q}_{h}\right)(\boldsymbol{x})\left(T-S_{h}\right)(\boldsymbol{x})(\boldsymbol{x}) d \boldsymbol{x} .
\end{aligned}
$$


All this gives

$$
\left\|T_{h}-S_{h}\right\|_{L^{2}(\Omega)} \leq c\left(\left\|\boldsymbol{p}-\boldsymbol{p}_{h}\right\|_{\mathbb{X}}+\left\|T-S_{h}\right\|_{L^{2}(\Omega)}\right),
$$

and we conclude by using the first estimate in (4.13) and a triangle inequality.

Evaluating the distance of $T$ to $\mathbb{M}_{h}$ relies on fully standard arguments, see [5, Chap. IX, Th. 2.1] for instance. On the other hand, we deduce from Lemma 4.3 that, for all $\boldsymbol{q}_{h}$ in $\mathbb{X}_{h}^{0}$,

$$
\left\|\boldsymbol{p}-\Pi_{h} \boldsymbol{p}\right\|_{\mathbb{X}} \leq\left\|\boldsymbol{p}-\boldsymbol{q}_{h}\right\|_{\mathbb{X}}+\left\|\Pi_{h}\left(\boldsymbol{p}-\boldsymbol{q}_{h}\right)\right\|_{\mathbb{X}} \leq c\left\|\boldsymbol{p}-\boldsymbol{q}_{h}\right\|_{\mathbb{X}}
$$

and we take $\boldsymbol{q}_{h}$ equal to the interpolate of $\boldsymbol{p}$ by piecewise affine functions, see [5, Chap. IX, Prop. 1.4].

We are in position to provide the error estimate. We need first to complete the notation. We define the space

$$
\begin{aligned}
\mathbb{X}^{s, r}=\{\boldsymbol{q} \in \mathbb{X} ; \quad & \left.(\boldsymbol{q}, \operatorname{div} \boldsymbol{q})\right|_{\Omega_{S}} \in H^{s}\left(\Omega_{S}\right)^{d+1} ; \\
& \left.\left.(\boldsymbol{q}, \operatorname{div} \boldsymbol{q})\right|_{\Omega_{G}} \in H^{r}\left(\Omega_{G}\right)^{d+1} ;\left.\quad(\boldsymbol{q} \cdot \boldsymbol{n})\right|_{\gamma} \in H^{\min \{s, r\}}(\gamma)\right\} .
\end{aligned}
$$

The following result is a direct consequence of Lemma 4.6.

Theorem 4.7. Assume that the solution $(T, \boldsymbol{p})$ of problem (2.11) is such that

$$
\boldsymbol{p} \in \mathbb{X}^{s, r}, \quad T \in H^{s}\left(\Omega_{S}\right) \times H^{r}\left(\Omega_{G}\right)
$$

for real numbers $s, r$ such that $0 \leq s, r \leq 1$. Then, the following a priori error estimate holds between this solution and the solution $\left(T_{h}, \boldsymbol{p}_{h}\right)$ of problem (4.3)

$$
\left\|\boldsymbol{p}-\boldsymbol{p}_{h}\right\|_{\mathbb{X}}+\left\|T-T_{h}\right\|_{L^{2}(\Omega)} \leq c h^{\min \{s, r\}}\left(\|\boldsymbol{p}\|_{\mathbb{X} s, r}+\left\|T_{S}\right\|_{H^{s}\left(\Omega_{S}\right)}+\left\|T_{G}\right\|_{H^{r}\left(\Omega_{G}\right)}\right) .
$$

Remark 4.8. Estimate (4.14) is fully optimal and proves the convergence of the discretization. Likely, super-convergence results as in Theorem 3.7 could be obtained on the field $\boldsymbol{p}$. Unfortunately we did not succeed in doing so. Nevertheless the numerical experiments we run show that this super-convergence takes place. 


\section{Numerical experiments.}

We describe some indicative numerical experiments for problem (1.2), in order to evaluate the ability of the Lagrange finite elements to produce accurate results for some simple geometries. We aim also to assess results stated in Section 3 and in particular the super-convergence provided in Theorem 3.7. Let us remark that to avoid any modification of the data structure of the finite element we resort to a domain decomposition method (see [14]). Then, we switch to investigation of the capacity of the Raviart-Thomas finite element method to provide reliable results in the hybrid dual formulation. We underline in several examples the pertinence of the convergence rate proved here. The computational study is conducted by means of the code freefem $\mathrm{em}_{++}$developed by F. Hecht and his team (see [12], [13]).

\subsection{An explicit solution.}

In the first example the domain $\Omega$ is the disc centered at the origin with radius 1.5 . The sub-domain $\Omega_{S}$ coincides with the unit circle and the sub-domain $\Omega_{G}$ is then the annulus with double radius $(1,1.5)$. The exact solution $T$ is given by

$$
\begin{aligned}
& T_{S}\left(x_{1}, x_{2}\right)=a\left(x_{1}^{2}-x_{2}^{2}\right) \\
& T_{G}\left(x_{1}, x_{2}\right)=\left(x_{1}^{2}-x_{2}^{2}\right)\left(b+\frac{c}{\left(x_{1}^{2}+x_{2}^{2}\right)^{2}}\right) .
\end{aligned}
$$
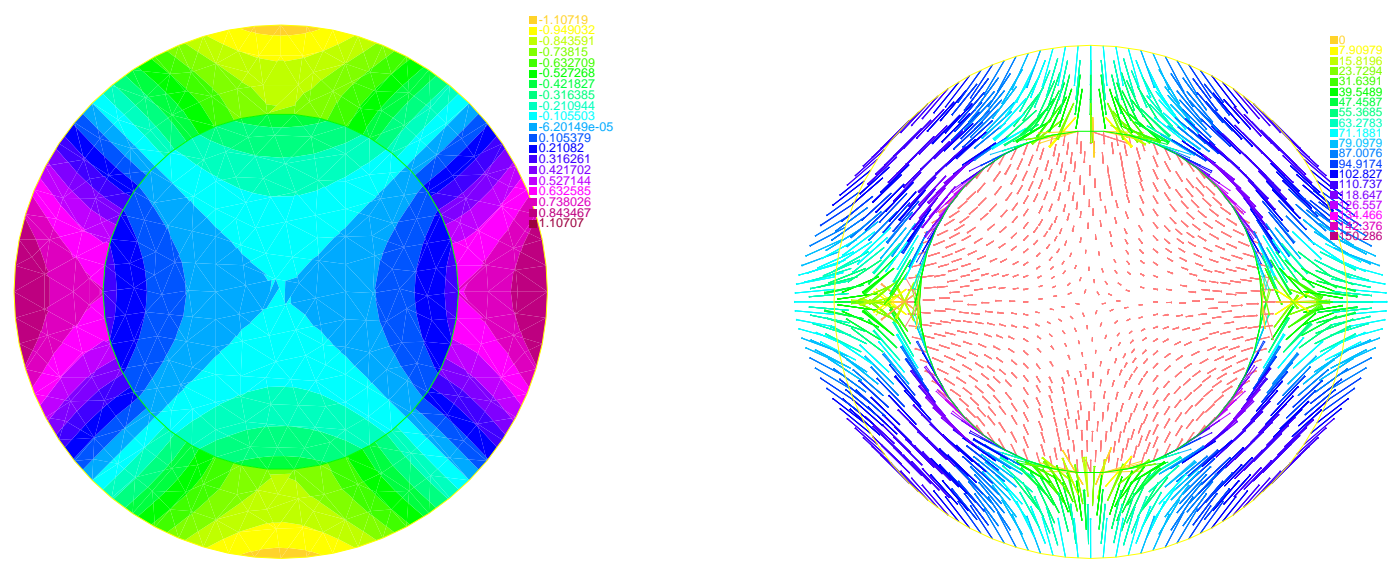

Figure 2 - Temperature field $T$. Heat vector field $\boldsymbol{p}$.

The conductivities are chosen so that $\left(\kappa_{S}, \kappa_{G}\right)=(1,100)$ and the contact resistivity is fixed to $R=0.5$. The coefficients $a, b, c$ are computed owing to the interface conditions so as the Dirichlet datum enforced on the whole boundary $\partial \Omega$ which is provided by

$$
T_{D}\left(x_{1}, x_{2}\right)=\frac{4}{9}\left(x_{1}^{2}-x_{2}^{2}\right)
$$


The temperature $T$ and the heat vector field $\boldsymbol{p}=\kappa \nabla T$ are represented in Figure 2 .

Next, we compute the discrete solutions $T_{h}$ by Lagrangian finite element method and $\left(T_{h}, \boldsymbol{p}_{h}\right)$ by hybrid dual finite element method, for various meshes. The gap with the exact $(T, \boldsymbol{p})$ is evaluated in the $L^{2}$-norm. In the Lagrange computations, the vector field $\boldsymbol{p}_{h}$ is not an independent unknown. It is indeed derived by computing the gradient of $T_{h}$ and is then piecewise constant. Figure 3 provides the errors for both methods in logarithmic scales. To check out the convergence rates for each method we need the slopes of the linear regressions of the error curves. In the Lagrange method, we found the slopes $(1.92,0.99)$ for the temperature $T$ and the heat vector field $\boldsymbol{p}$. Those in the dual hybrid method are given by $(1.19,1.04)$. Given that the effective smoothness of the exact temperature is higher than $H^{2}\left(\Omega_{S}\right) \times H^{2}\left(\Omega_{G}\right)$, the convergence speed is hence limited only by the degree of the finite elements we use. This is in agreement with the theoretical predictions in Theorem 3.2, Proposition 3.4 and Theorem 4.7.
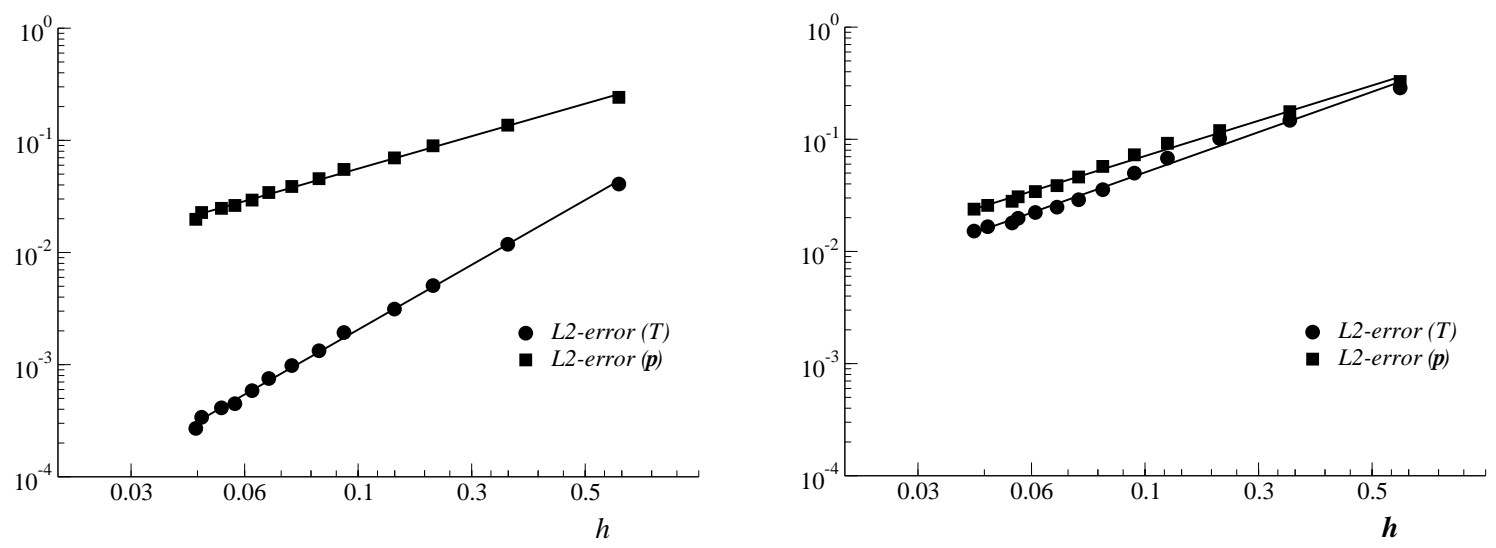

Figure 3 - Convergence curves. Lagrange FEM (left) and hybrid dual FEM (right).

\subsection{A singular solution.}

In the second experiment we pursue an approximation of a singular solution. The domain $\Omega$ is a disc centered at the origin with radius 0.5 . The internal sub-domain $\Omega_{S}$ is a triangle. The geometry can be seen in Figure 4 . The circular boundary $\partial \Omega$ is subjected to the following Dirichlet condition (here also $\Gamma_{N}=\emptyset$ )

$$
T_{D}\left(x_{1}, x_{2}\right)=2 e^{2\left(x_{1}-x_{2}\right)} \cos \left(\frac{\pi}{2}\left(x_{1}+x_{2}\right)\right) .
$$

The thermal parameters are unchanged compared with the first test, that is $\kappa_{G}=100, \kappa_{S}=$ 1 and $R=0.5$. The isolines of the solution are represented in Figure 4.

Following the theoretical discussion in Section 3.2, only $\Omega_{G}$ is expected to suffer from the singularities born at the vicinity of the vertices of $\Omega_{S}$. The convergence rate (in $\Omega_{G}$ ) 
is lower than one for the heat vector field $\boldsymbol{p}$. It is expected to be equal to $4 / 7$ or may be to $2 / 3$ if the singularity created by the right angle is more intensive than the two others. A careful examination of the heat vector field plot provided in Figure 4 seems to corroborate this fact. The stress intensity factor at the right angle is substantially higher than for the two acute angles. The accuracy in the convex set $\Omega_{S}$ should not slow down and the decreasing rate is excepted to be linear here again.
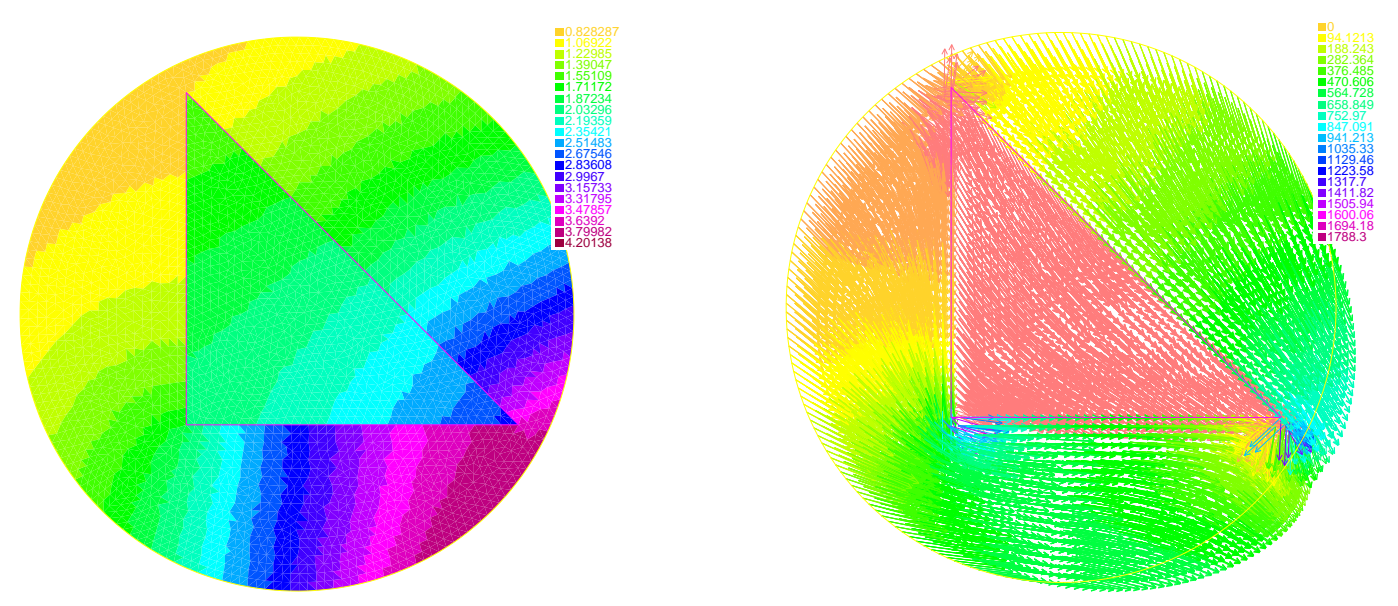

Figure $4-$ Singular temperature $T$. Singular heat vector field $\boldsymbol{p}=\kappa \nabla T$.

Now, to check out these claims, we compute a reference finite element solution $\left(T_{h}, \boldsymbol{p}_{h}\right)$ using a high resolution mesh and assimilate it to the exact $(T, \boldsymbol{p})$. Then, we run numerical simulations using meshes with moderate sizes. The slopes of linear regressions of the convergence curves are $(1.93,0.97)$ for $(T, \boldsymbol{p})$ in the internal sub-domain $\Omega_{S}$ and $(1.52,0.70)$ in the sub-domain $\Omega_{G}$. The convergence in $\Omega_{S}$ seems to be of order one for $\boldsymbol{p}$ and of a second order for $T$. The lower order convergence observed in $\Omega_{G}$ is an illustration of the effectiveness of the angular singularity which is responsible of the slowing down of the convergence speed. These trends are almost in perfect agreement with the theoretical findings concerning the convergence for $\boldsymbol{p}$. Concerning the special behavior of the error on $T$ in the hybrid method, we fall short in the proof of the observed convergence rate. This is still an open question. 

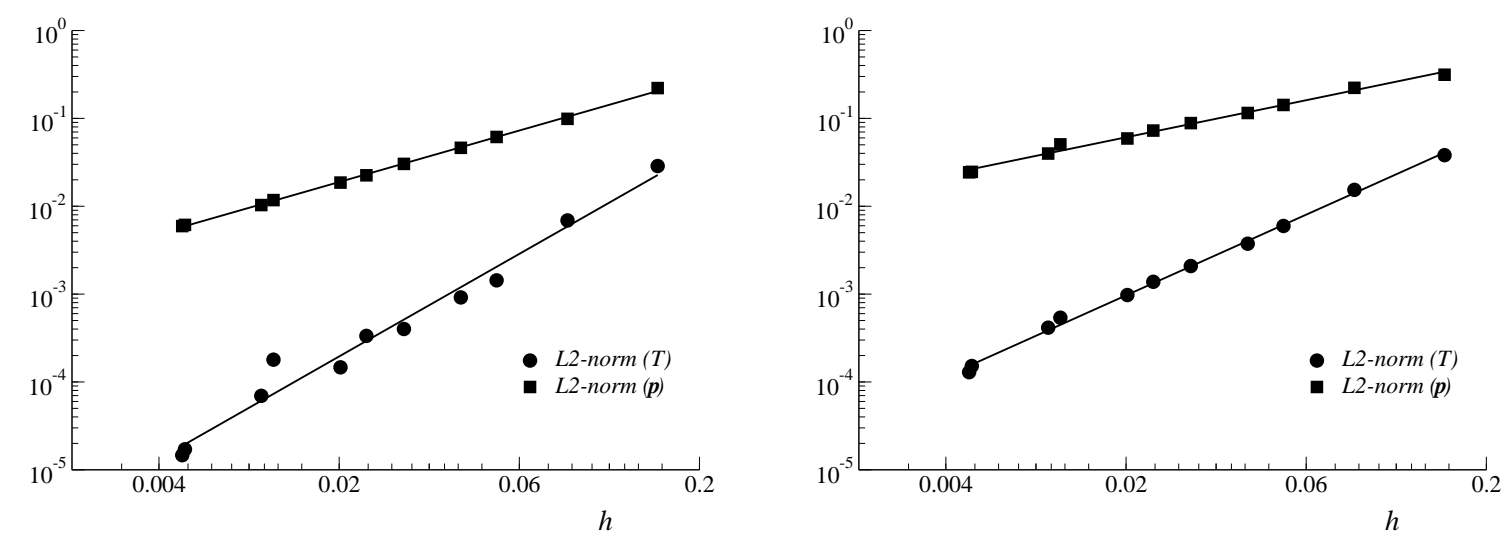

Figure 5 - Accuracy curves for the Lagrange FEM in $\Omega_{S}$ (left) and in $\Omega_{G}$ (right).

Switching to the hybrid dual solutions, the convergence rates are all expected to be linear except for the heat field $\boldsymbol{p}$ in the external sub-domain $\Omega_{G}$. The slope for the linear regression of the accuracy curve for $\left.\boldsymbol{p}\right|_{\Omega_{G}}$ is close to 0.64 . The convergence rates evaluated in $\Omega_{S}$ give $(0.96,1.01)$ for $(T, \boldsymbol{p})$.
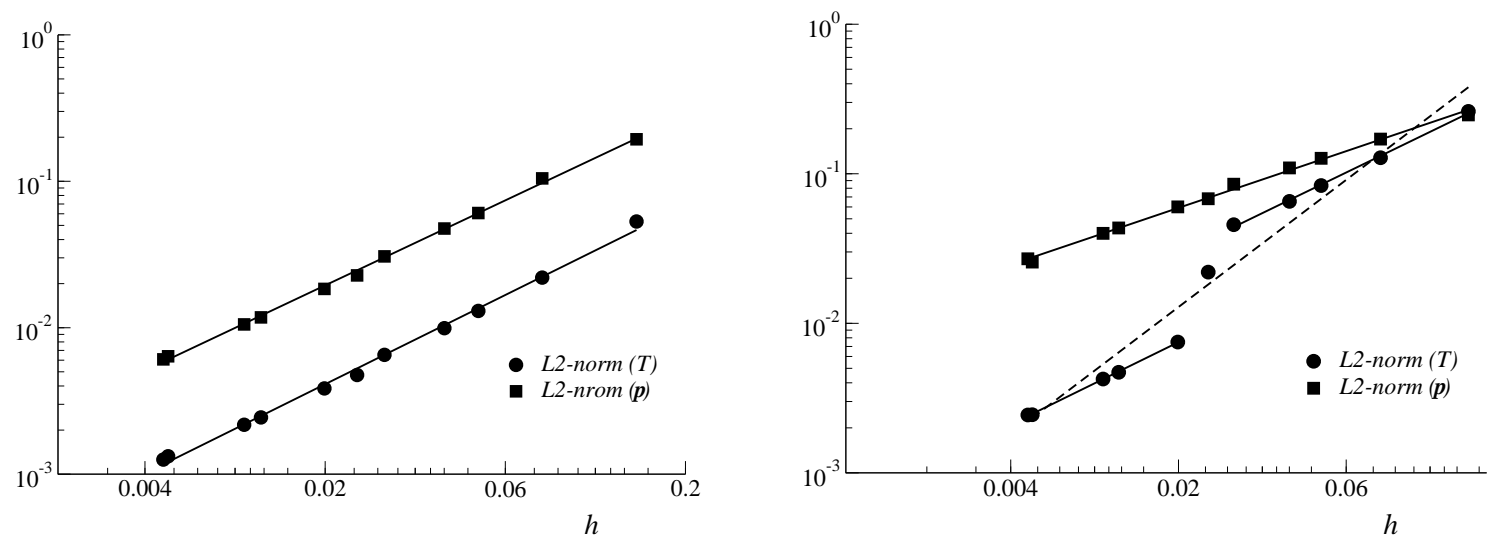

Figure 6 - Accuracy curves for the hybrid dual FEM in $\Omega_{S}$ (left) and in $\Omega_{G}$ (right).

Finally, the curve for the $L^{2}$-error on $T$ in $\Omega_{G}$ seems a little bit strange. The apparent slope of the linear regression (dashed line) is 1.41 and it does not seem pertinent. However a closer look to that curve shows that it has separate components, the slope of each is not far form one as we have 0.90 a for the first component and 0.91 for the last one. We unfortunately have no explanation of this break off in the convergence curve. None of the observations made here on the super-convergence in the internal sub-domain $\Omega_{S}$, is mathematically proved. Nevertheless, they are in a perfect accordance with the common feeling. 


\subsection{A realistic geometry.}

We conclude with an example of a realistic geometry depicted in Figure 1, right part. As indicated earlier running computations can be reasonably achieved for the hybrid dual method. The composite medium we consider is a small sample that has a rectangular shape. The width is equal to 0.003222 while the length is 0.005382 . Almost a fraction four fifths of the media is made of salt which corresponds to the yellow part of the domain in Figure 1. The remaining one fifth fraction is composed of graphite and is colored in red. The sample is differentially heated along horizontal walls. The temperature is then fixed to $T_{D}=587$ along the upper wall. It is given by $T_{D}=577$ along the lower wall. Both vertical walls are adiabatic. The conductivities are chosen to be $\kappa_{S}=1$ and $\kappa_{G}=500$. They are close to the real conductivities of the graphite and of the sodium chloride. We realized two simulations. In one the resistance is given by $R=5 \times 10^{-4}$ and in the other it is equals $R=5 \times 10^{-2}$. The temperature fields are depicted in Figure 7. For larger resistance the diffusion process of the heat from the upper wall toward the lower wall is somehow slowed down, especially in the right side of the sample. Indeed, the heat has to flow across more interfaces there than in the left side of the sample. These computations bring to light the efficiency of the discretization that we propose.
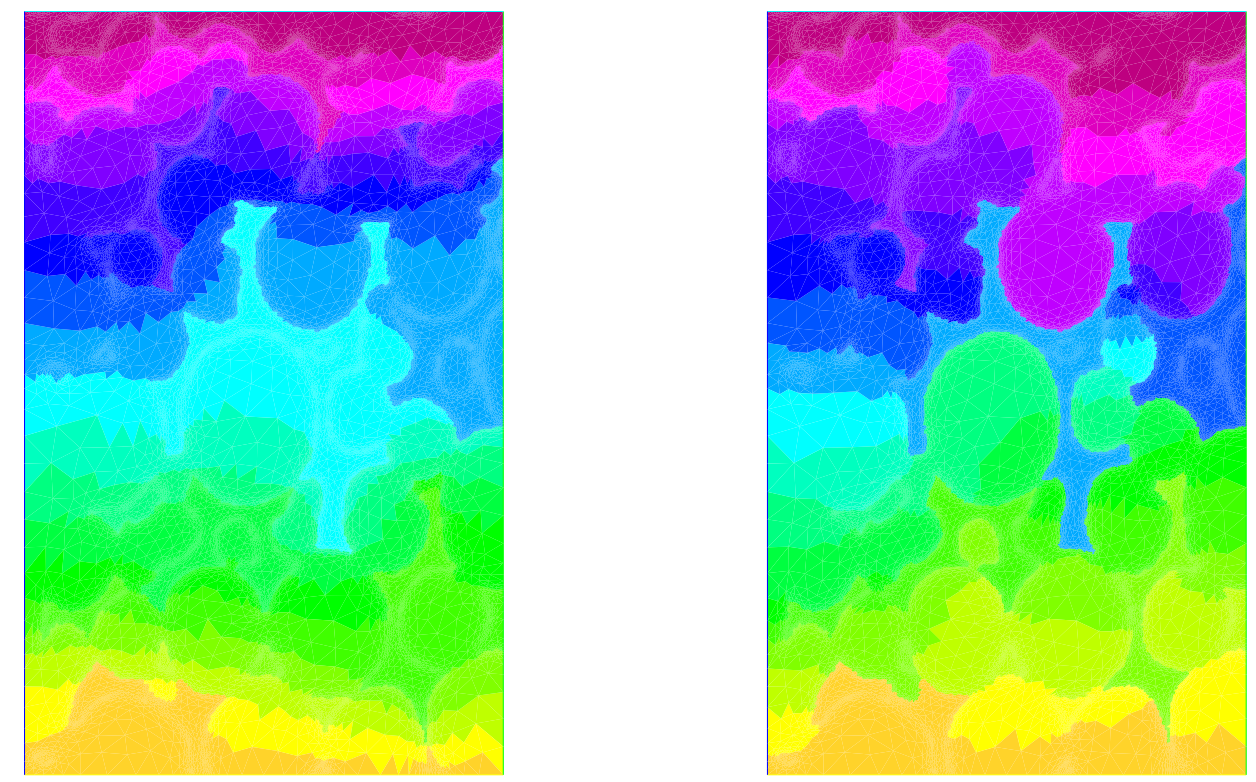

Figure 7 - Two simulations for a realistic domain. 


\section{Conclusion.}

Hybrid dual finite elements and alike methods sound well fit for the numerical simulation of the heat diffusion in composite media with contact resistance. The reason why we undertook this work is the feasibility and implementation facility. Using hybrid dual finite elements enables users to build their simulations on existing data structures in softwares. The action of programmers is limited to the definition of the variational problem to solve. Things are obviously different for Lagrangian finite elements especially for complex geometry such as the one considered in the third example of the numerical section. Indeed, the finite element structure in scientific computing codes has to be revisited and drastically modified to account for the local continuity/global discontinuity. The numerical analysis conducted here shows the reliability of the mixed Raviart-Thomas/piecewise constant finite elements $R T_{0} / \mathcal{P}_{0}$ to provide an accurate discrete solution to the heat problem (1.2). 


\section{References}

[1] R.A. Adams, J. Fournier — Sobolev Spaces, Academic Press, 2003.

[2] D. N. Arnold, F. Brezzi, B. Cackburn, L. D. Marini - Unified analysis of discontinuous Galerkin methods for elliptic problems. SIAM J. Numer. Anal. 39 (2002), 1749-1779.

[3] J.-M. Bernard - Density results in Sobolev spaces whose elements vanish on a part of the boundary, Chinese Annals of Math., Ser. B 32 (2011), 823-846.

[4] C. Bernardi, Y. Maday, A.T. Patera - A new nonconforming approach to domain decomposition: the mortar element method, Collège de France Seminar, H. Brezis and J.-L. Lions eds., Pitman (1990).

[5] C. Bernardi, Y. Maday, F. Rapetti - Discrétisations variationnelles de problèmes aux limites elliptiques, Collection "Mathématiques et Applications" 45, Springer-Verlag (2004).

[6] S. Brenner, L. R. Scott — The Mathematical Theory of Finite Element Method, Texts in Applied Mathematics 15, Springer (2008).

[7] F. Brezzi, M. Fortin — Mixed and Hybrid Finite Element Methods, Springer-Verlag (1991).

[8] P.-G. Ciarlet - The Finite Element Method for Elliptic Problems, North Holland, Amsterdam (1978).

[9] L. S. Fletcher - Conduction in solids, imperfect metal-to-metal contacts: Thermal contact resistance, Section 502.5, Heat Transfer and Fluid Mechanics Data Books, Genium Publishing Company, Schenectady, New York (1991).

[10] V. Girault, P.-A. Raviart - Finite Element Methods for Navier-Stokes Equations, Theory and Algorithms, Springer-Verlag (1986).

[11] P. Grisvard - Elliptic Problems in Nonsmooth Domains, Pitman (1985).

[12] F. Hecht - Freefem ++ . Third Edition, Version 3.30, http://www.freefem.org/ff++.

[13] F. Hecht - New development in freefem++. J. Numer. Math. 20 (2012), 251-265.

[14] F. Jelassi, M. Azaïez, E. Palomo Del Barrio - A substructuring method for phase change modelling in hybrid media. Computers \& Fluids 88 (2013), 81-92.

[15] D. Jerison, C. E. Kenig - The Neumann problem on Lipschitz domains, Bull. Amer. Math. Soc. 4 (1981), 203-207.

[16] J.-L. Lions, E. Magenes - Problèmes aux limites non homogènes et applications, Vol. I, Dunod, Paris (1968).

[17] P.-A. Raviart, J.-M. Thomas - A mixed finite element method for second order elliptic problems, in Mathematical Aspects of Finite Element Methods, Lecture Notes in Math., Vol. 606, pp. 292-315, Springer (1977).

[18] J. E. Roberts, J.-M. Thomas - Mixed and Hybrid Methods, Handbook of Numerical Analysis, P.G. Ciarlet and J.-L. Lions eds., vol. II, Finite Element Methods (Part I), pp. 523-639, Elsevier Science Publishers, Amsterdam (1991).

[19] Y. Safa - Simulation numérique des phénomènes thermiques et magnétohydrodynamiques dans une cellule de Hall-Héroult. Ph. D. Ecole Polytechnique Fédérale de Lauzanne (2005).

[20] E. T. Swartz, R. O. Pohl - Thermal boundary resistance, Rev. Mod. Phys. 61, 605 (1989). 